\title{
Labyrinthe
}

31 | 2008 (3)

Revues modes d'emploi

\section{Le corps, le ghetto et l'État pénal}

Entretien réalisé par Susana Durão

Loïc Wacquant

\section{(2) OpenEdition}

Journals

Édition électronique

URL : http://journals.openedition.org/labyrinthe/3920

DOI : $10.4000 /$ labyrinthe.3920

ISSN : 1950-6031

Éditeur

Hermann

Édition imprimée

Date de publication : 15 octobre 2008

ISBN : 978-2-9526131-8-7

Référence électronique

Loïc Wacquant, « Le corps, le ghetto et l'État pénal », Labyrinthe [En ligne], 31 | 2008 (3), mis en ligne le 23 février 2009, consulté le 19 avril 2019. URL : http://journals.openedition.org/labyrinthe/3920 ; DOI : 10.4000/labyrinthe.3920

Propriété intellectuelle 


\section{Entretien}

\section{LE CORPS, LE GHETTO ET L'ÉTAT PÉNAL ${ }^{*}$}

\section{Sur le terrain avec Lö̈ Wacquant}

Résumé : Dans cet article, l'auteur explicite son approche de l'ethnographie, de la théorie sociale et de la politique du savoir, à travers un entretien au fil duquel il démêle les liens entre ses travaux sur l'incorporation, la marginalité urbaine et l'État pénal. En retraçant son parcours intellectuel, il porte au jour les connexions pratiques et épistémologiques entre ses principaux projets de recherche, spécifie la contribution du travail de terrain à chacun d'eux, et examine les rôles des intellectuels dans les sociétés avancées à l'ère de l'hégémonie néolibérale. Renvoyant dos à dos l'empirisme de Hume et le cognitivisme néo-kantien, l'auteur défend l'usage de l'ethnographie comme instrument de rupture et de construction, souligne la puissance des compétences incarnées, et plaide pour l'impératif de réflexivité épistémique ainsi que pour la multiplication des genres textuels et des styles d'écriture afin de mieux capturer la douleur et la saveur de l'action. Dans la sphère publique, il argue que la science sociale est en mesure d'agir comme dissolvant de la doxa mais aussi comme phare éclairant les propriétés latentes et les tendances inaperçues des transformations sociales, de sorte à perturber et à élargir le débat civique.

Mots-clés : ethnographie, théorie, épistémologie, réflexivité, Pierre Bourdieu, ghetto, marginalité, état pénal, intellectuels, politiques du savoir, néolibéralisme

Abstract : This article dissects the author's approach to ethnography, social theory, and the politics of knowledge through a dialogue retracing his intellectual trajectory and the analytic linkages between his inquiries

\footnotetext{
* Une version abrégée de cet article est parue sous format papier dans Labyrinthe, $\mathrm{n}^{\circ} 31$, 2008. Toute citation opérée à partir de la version longue ici sous forme de PDF doit porter les références de la version électronique disponible sur le site Web de la revue.
} 


\section{Labyrinthe, $n^{\circ} 31$}

into embodiment, comparative urban marginality and the penal state. It draws out the practical connections and epistemological rationale behind his main research projects, explicates the distinctive ways in which he deploys observational fieldwork in each of them, and examines the roles of intellectuals in advanced society in the era of hegemonic neoliberalism. Rejecting both Humean empiricism and neoKantian cognitivism, the author argues for the use of ethnography as an instrument of rupture and construction, the potency of carnal knowledge, the imperative of epistemic reflexivity, and the need to expand textual genres and styles so as to better capture the taste and ache of social action. In the public sphere, he proposes that social science can act as a solvent of doxa and a beacon casting light on latent properties and unnoticed trends in social transformations so as to disrupt and broaden civic debate.

Keywords : ethnography, theory, epistemology, reflexivity, Pierre Bourdieu, ghetto, marginality, penal state, intellectuals, politics of knowledge, neoliberalism

Un sociologue peut-il parler de lui, son parcours et ses travaux, sans tomber dans les pièges du subjectivisme ? C'est le pari de Loïc Wacquant, professeur à l'Université de Californie à Berkeley et membre du Centre de sociologie européenne, dans l'entretien qu'il a accordé à Susana Durão lors des rencontres de l'Ethnografeast III, à Lisbonne en juin $2007^{* *}$. Guidé par le souci réflexif qu'il place au cœur de sa démarche, le sociologue y revient sur la rencontre fondatrice avec Pierre Bourdieu, ses premiers travaux en Nouvelle-Calédonie, les hasards socialement structurés qui décident d'une carrière, et les pères spirituels qu'il s'est choisis en passant d'une discipline (et d'un continent) à l'autre. Surtout, l'auteur de Les Prisons de la misère (Raisons d'agir, 1999) et Corps et âme. Carnets ethnographiques d'un apprenti boxeur (Agone, Comeau \& Nadeau, 2000), ou encore de Parias urbains. Ghetto, banlieues, État (La

\footnotetext{
${ }^{* *}$ Entretien publié, en portugais, dans la revue Etnografica (Lisbonne, automne 2008), et en anglais dans la revue Qualitative Sociology (Austin, Texas, hiver 2008). La plupart des textes de Loïc Wacquant cités ci-après sont accessibles en en ligne sur son site personnel : http://sociology.berkeley.edu/faculty/wacquant [site consulté le 30 août 2008]. Une version réduite de cet entretien figure dans Labyrinthe, $n^{\circ} 31,2008$ (3).
} 


\section{Le corps, le ghetto et l'État pénal}

Découverte, 2006), également co-auteur avec Pierre Bourdieu de An Invitation to Reflexive Sociology (traduit en français en version abrégée sous le titre Réponses. Pour une anthropologie réflexive, Seuil, 1992), détaille ici comment s'articulent ses recherches sur le ghetto noir aux États-Unis, la pratique de la boxe comme métier du corps et la transition « de l'État pénal à l'État social » à l'ère du salariat fragmenté, en revenant notamment sur ses protocoles méthodologiques et ses modes d'écriture, novateurs dans les sciences humaines. Autant de réflexions à la charnière des disciplines, à la frontière entre le politique et le savant, et qui, se nourrissant d'une position géographique à cheval sur deux continents, trouvent très naturellement leur place dans Labyrinthe.

Le texte qui suit s'appuie sur un entretien réalisé à Lisbonne, au Portugal, lors de la troisième édition de l'Ethnografeast, qui s'est tenue au Centre d'Etudes d'Anthropologie Sociale (ISCTE) sous les auspices de la revue Ethnography, du 20 au 23 juin 2007 (avec le soutien des fondations Wenner-Gren, Lusitano-américaine et Calouste Gulbenkian; pour en savoir plus les conférences elles-mêmes, voir : http://ceas.iscte.pt/ethnografeast). Lors de ces rencontres, j'ai pu m'asseoir en compagnie de Loïc Wacquant afin de discuter de son approche de l'ethnographie, de la théorie en sciences sociales, et du débat civique. La majorité de ses textes (dont plus d'une centaine d'articles et une demi-douzaine de livres) ont d'abord été publiés aux États-Unis ou en France, mais ont rapidement été traduits, lus et discutés dans les diverses sciences sociales sur quatre continents.

Ce dialogue retrace la trajectoire intellectuelle de Wacquant et offre une cartographie de ses enquêtes de terrain, centrée sur la triade de l'incorporation, l'analyse comparative de la marginalité urbaine et l'État pénal, débouchant sur une réflexion sur les rapports entre savoir et pouvoir(s). Il sonde les fondements pratiques et épistémologiques de ces projets de recherche divers, décortique les usages qu'il fait du travail de terrain dans chacun d'eux, et explore le rôle des intellectuels dans les sociétés avancées à l'âge de l'hégémonie néolibérale. (Les références bibliographiques orientent le lecteur vers ses textes clés pour chaque point abordé; le texte original en portugais est à paraître en deux parties dans Etnografica et une version anglaise dans Qualitative Sociology).

Susana Durão (ISCTE, Lisboa) 
Susana Durão - J'aimerais profiter de votre présence à Lisbonne, dans le cadre de ces stimulantes rencontres de l'Ethnografeast III sur "Ethnographie et sphère publique 》 - que vous avez organisées avec Manuela Ivone Cunha et Antónia Pedroso de Lima - pour retracer brièvement l'histoire de cette "fête", et aussi pour que vous nous donniez une idée de votre parcours académique assez singulier.

Loïc Wacquant - Je suis très heureux que le Portugal accueille les troisièmes rencontres de l'«Ethnografeast », après Berkeley en 2002 et Paris en 2004, organisées sous l'égide la revue Ethnography. Comme son nom l'indique, et conformément à la ligne intellectuelle de la revue, l'Ethnografeast est une sorte de célébration collective de l'ethnographie par ceux qui s'y consacrent dont l'objet est à la fois ludique, pratique et scientifique ${ }^{1}$. Il s'agit d'abord de créer de l' " effervescence collective ", comme aurait dit Émile Durkheim, pour renouveler nos énergies et notre engagement dans le travail de terrain, et surtout encourager les jeunes chercheurs à s'y investir - c'est pourquoi l'édition de Lisbonne consacre une journée entière aux travaux des doctorants et des ethnographes de la nouvelle génération.

C'est ensuite l'occasion d'activer le dialogue entre les disciplines qui pratiquent l'ethnographie (en commençant par la sociologie et l'anthropologie mais en allant bien au-delà), mais aussi entre les différents genres de l'ethnographie, entre les traditions théoriques qui l'informent, entre les générations et entre les continents et les pays : nous avons réuni à Lisbonne des chercheurs venant des États-Unis, de France, d'Italie, d'Angleterre, de Hollande, du Brésil, d'Afrique du Sud et bien sûr d'Espagne et du Portugal... Certains pensent que l'ethnographie est en crise, d'autres qu'elle est en plein boom; les uns y voient une pratique essentiellement herméneutique et littéraire, les autres un outil de preuve scientifique ou de construction théorique, d'autres encore une forme de la conscience collective des sociétés contemporaines - bref une très grande variété de styles et de positions vont se confronter. L'idée est d'ouvrir au maximum le compas des débats, et de ce point de vue, Manuela et Antónia, à qui revient tout le mérite de cette rencontre, ont magnifiquement rempli le cahier des

\footnotetext{
${ }^{1}$ Loï Wacquant, «Ethnografeast : A Progress Report on the Practice and Promise of Ethnography », Ethnography, 4-1, mars 2003, p. 1-10. Comme bon nombre des textes de Loïc Wacquant cités ci-dessous, celui-ci est disponible, en ligne, sur son site personnel : http://sociology.berkeley.edu/faculty/wacquant [site consulté le 30 août 2008].
} 
charges. Enfin, l'Ethnografeast a pour but de nous aider à élaborer et à clarifier collectivement les paramètres et les missions de l'ethnographie dans la cité savante comme dans le débat civique et politique. C'est d'ailleurs le thème qui anime nos rencontres à Lisbonne : «Ethnographie et Sphère Publique ».

\section{Du Sud de la France Au PACIFIQUe Sud}

Susana Durão - Venons-en à votre itinéraire alors. Vous avez commencé par être l'élève de Pierre Bourdieu, avec qui vous avez ensuite travaillé pendant près de vingt ans. Pouvez-vous retracer le cheminement personnel et intellectuel qui vous a amené à le rencontrer?

Loïc Wacquant - Je suis né dans le sud de la France, dans une famille de la classe moyenne intellectuelle et $\mathrm{j}$ 'ai fait mes études à l'école publique de mon village puis au grand lycée de la ville voisine, Montpellier. Ensuite je suis monté à Paris où, ne sachant pas trop quel cursus suivre, j'ai d'abord étudié l'économie industrielle. Je suis entré dans une grande école de gestion, l'École des HEC, à Paris, par défaut plus que par vocation : je n'étais pas assez « matheux » pour être attiré par Polytechnique et pas assez «littéraire» pour envisager l'École normale Supérieure, donc j'avais choisi un concours dont le profil tombait entre les deux. J'avais en tête de faire de l'économie politique mais j'ai dû vite déchanter: HEC est une école professionnelle qui vous prépare à être manager dans une grande entreprise, et j'étais horrifié à cette idée. Je cherchais donc à virer de bord et j'envisageais de faire de l'histoire sociale (un de mes livres préférés à ce moment était Louis XIV et quinze millions de français, une étude-type de l'École des Annales par Pierre Goubert ${ }^{2}$ ) quand, un soir, un ami m'a amené assister à une conférence de Pierre Bourdieu sur le thème «Questions de politique ». C'était en novembre 1980, juste après la sortie du Sens pratique et avant sa nomination au Collège de France ${ }^{3}$. Pour moi cette conférence a été une vraie révélation: je n'ai pas

\footnotetext{
${ }^{2}$ Pierre Goubert, Louis XIV et vingt millions de français, Paris, Hachette, 1997 (1967). ${ }^{3}$ Pierre Bourdieu, Le Sens pratique, Paris, Minuit, 1980; le type et le style de conférence publique que Bourdieu donnait à l'époque est bien restitué dans Questions de sociologie, Paris, Minuit, 1980.
} 
compris les trois-quarts de ce que Bourdieu racontait mais j'ai bien saisi que quelque chose de très important se disait et qu'il fallait le creuser.

Susana Durão - Quel âge aviez-vous?

Loïc Wacquant - J'avais juste vingt ans. Après la conférence, nous avons eu une discussion passionnante avec Bourdieu dans la cafétéria des étudiants, jusqu'à quatre heures du matin. J'ai eu le sentiment très vif, en l'écoutant répondre à nos questions tous azimuts, que, tel un chirurgien, il découpait le corps de la société française pour nous en montrer les entrailles et le fonctionnement interne d'une manière que je n'aurais jamais cru possible. En rentrant de cette conférence au petit matin, je me suis dit : «Si c'est ça la sociologie, c'est ça que je veux faire $»$.

Mais si cette conférence a marqué un déclic, c'est sans doute que j'avais des dispositions en ce sens en raison de ma trajectoire familiale et personnelle. J'avais acquis un ail proto-sociologique, du fait de la mobilité sociale de mes parents, qui avait fortement marqué ma petite enfance, des tiraillements de classe dans le village où j'ai grandi, et du fait aussi de ma mobilité géographique et régionale. Venant du sud, habiter en lisière de Paris, c'était quasiment s'expatrier! Au final, je suis redevable de mon expérience à HEC, même si je m'y suis terriblement ennuyé, parce que ça m'a mis en contact avec un monde, celui de l'entreprise, dans lequel j'ai découvert que je ne voulais pas aller et que j'ai fui pour aller vers l'univers de la recherche. Ensuite ma scolarité sur ce campus m'a fait me poser des tas de questions et m'a indirectement poussé vers la sociologie par le choc culturel frontal qu'était pour moi le fait me trouver immergé au milieu des enfants de la haute bourgeoisie parisienne et de la noblesse - dont je croyais, naïvement, qu'on l'avait éliminée en 1789 ! Mon voisin de chambre s'appelait Christian de Rivelrieux de Varax et jouait du cor de chasse le soir à notre balcon commun, c'est vous dire...

Encouragé par cette rencontre avec Bourdieu, j'ai entamé, parallèlement à mes études d'économie industrielle, un cursus universitaire en sociologie. J'ai fait ma licence puis ma maîtrise à Nanterre - qu'on surnommait encore à l'époque «Nanterre la rouge $»$ ! Participer à ces deux univers en même temps, une grande école vouée à la perpétuation des milieux d'affaires parisiens et une université publique creuset historique de la subversion étudiante et de la critique sociale, c'était une bonne introduction pratique à la 


\section{Le corps, le ghetto et l'État pénal}

sociologie. Sur le campus d'HEC, j'étais un élève dissident à plusieurs titres, politique et pédagogique, rebelle à l'endoctrinement grossier qu'on subissait - je me souviens de citer La Reproduction et même Le Système des objets de Baudrillard ${ }^{4}$, en cours de marketing pour provoquer le prof! Nous étions un petit groupe où se retrouvaient les rares élèves issus des classes populaire et moyenne et presque tous les provinciaux qui, en général, étaient de gauche, intellectuels et engagés. On nous appelait « les bolchos» et nous, nous appelions les tenants de l'ordre scolaire et social établi «les fachos ». C'était assez badin, sauf pendant les élections de 1981 où les relations se sont fortement tendues...

Susana Durão - Comment vous êtes-vous retrouvé en NouvelleCalédonie puis aux États-Unis?

Loïc Wacquant -A la sortie d'HEC, j'ai obtenu une bourse doctorale pour aller aux États-Unis, où j'ai passé une année studieuse en 198283 à Chapel Hill, à l'Université de Caroline du Nord. C'est là que s'est confirmée ma conversion de l'économie vers la sociologie. J'ai lu avec voracité (parmi mes livres favoris, ceux d'Elliott Liebow, John Dollard, C. Vann Woodward et Erving Goffman) et suivi des cours de théorie sociologique et de sociologie historique et comparative dans un excellent département, où je me suis lié d'amitié intellectuelle avec Gerhard Lenski et Craig Calhoun, qui m'ont encouragé dans ma mue. Tous les jeudis pendant un semestre, je déjeunais dans son bureau avec Lenski (l'auteur du classique Power and Privilege) et nous bavardions à bâtons rompus de théorie et d'histoire ${ }^{5}$. Ensuite je suis parti deux ans en Nouvelle-Calédonie en 1983-85 pour y faire mon service militaire, mais dans le cadre de l'assistance technique. Par une chance inouïe, c'était un service civil en tant que sociologue dans un centre de recherche de l'ORSTOM, l'ancien "office de recherche coloniale » de la France. Cela m'a donné deux années de formation à la pratique sociologique dans un contexte épineux et donc particulièrement instructif.

\footnotetext{
${ }^{4}$ Pierre Bourdieu \& Jean-Claude Passeron, La Reproduction. Éléments d'une théorie du système d'enseignement, Paris, Minuit, 1970 ; Jean Baudrillard, Le Système des Objets, Paris, Gallimard, 1968.

${ }^{5}$ Gerhard Lenski, Power and Privilege: A Theory of Social Stratification, Chapel Hill, University of North Carolina, [1968], 1984.
} 
À Nanterre, j'avais fait «Sociologie de la culture et de l'éducation » et écrit un mémoire de maîtrise mélangeant histoire et ethnographie, basé sur mon expérience à HEC, qui s'intitulait « Production scolaire et reproduction sociale », donc j'avais bien sûr lu et utilisé les travaux de Pierre Bourdieu. Durant ma dernière année à Paris, je séchais mes classes à HEC pour assister à son cours du Collège de France. Après chaque séance, Bourdieu et moi marchions ensemble jusque chez lui en discutant - pour moi, c'était comme un cours particulier accéléré! Et quand je suis parti en NouvelleCalédonie, nous avons entamé une correspondance suivie puis, après mon retour, j’ai été associé au Centre de sociologie européenne comme «membre expatrié ».

J'ai passé deux ans en Nouvelle-Calédonie, dans une toute petite équipe, puisqu'on était trois chercheurs, au moment du soulèvement kanak de novembre 1984. J'ai ainsi vécu et travaillé dans une société coloniale archaïque très brutale, puisque la Nouvelle-Calédonie, dans les années 1980, était une colonie type fin du XIX ${ }^{\mathrm{e}}$ siècle qui avait survécu, quasi-intacte, à la fin du $\mathrm{XX}^{\mathrm{e}}$. C'était une expérience sociale extraordinaire pour un apprenti-sociologue, que de mener des enquêtes sur le système scolaire, l'urbanisation et le changement social dans ce contexte d'insurrection, sous état d'urgence, d'observer en temps réel les luttes entre colons et indépendantistes, et de devoir réfléchir concrètement sur le rôle civique de la science sociale. J'ai ainsi participé à un congrès à huis clos du Front de Libération Nationale Kanak et Socialiste à Canala, fait le tour de la «Grande terre » (l'île principale), séjourné à plusieurs reprises à Lifou chez des amis militants kanak alors que pratiquement personne ne circulait sur le territoire.

C'est là aussi que j'ai lu les classiques de l'ethnologie, Mauss, Mead, Malinowski, Radcliffe-Brown, Bateson, etc. (notamment les travaux sur le Pacifique Sud : les îles Trobiands étaient juste à côté) et tenu mes premiers carnets de terrain (le tout premier a été griffonné dans la tribu de Luecilla, sur la baie de Wé, lors de la Noël 1983). Et publié mes premiers travaux, même pas «de jeunesse» mais d'enfance pourrait-on dire ${ }^{6}$. À la fin de mon séjour calédonien, j'ai

\footnotetext{
${ }^{6}$ Loïc Wacquant, «La question scolaire en Nouvelle-Calédonie: idéologies et sociologie ", Les Temps modernes, 464, mars 1985, p. 1654-85 [en ligne à l'adresse : http://horizon.documentation.ird.fr/exl-doc/pleins textes/pleins textes $5 / \mathrm{b}$ fdi 14 15/17302.pdf, page consultée le 30 août 2008] ; «Jeunesse, ordre coutumier et identité
} 


\section{Le corps, le ghetto et l'État pénal}

obtenu une bourse de quatre ans pour aller faire mon doctorat à l'Université de Chicago, berceau de la sociologie étasunienne. En arrivant dans la ville d'Upton Sinclair, mon intention était de travailler à une anthropologie historique de la domination coloniale en Nouvelle-Calédonie. Et puis j'ai été dérouté vers l'Amérique.

\section{LE GHETTO, LE GYM ET LA BANLIEUE}

Susana Durão - C'est ainsi qu'un jeune chercheur français va se frotter au ghetto noir américain...

Loïc Wacquant - En fait, deux événements imprévus se sont combinés. D'un côté, la porte néo-calédonienne s'est brusquement fermée : à Nouméa, le bureaucrate médiocre qui me chapeautait avait abusé de son autorité pour co-signer contre mon gré une monographie sur le système scolaire que j'avais réalisé seul - ce qui, tristement, était une pratique courante à l'ORSTOM ${ }^{7}$. J'ai dénoncé cette malversation intellectuelle auprès de la direction de l'Institut à Paris, qui évidemment s'est empressée de couvrir le fraudeur. Résultat, je me trouvais « interdit de séjour » dans cet organisme et, partant, sur l'île. De l'autre côté, je me suis trouvé confronté au quotidien à la réalité du ghetto de Chicago. J'habitais en lisière du quartier noir et pauvre de Woodlawn, et c'était un choc terrible d'avoir sous ma fenêtre ce paysage urbain quasi-lunaire, invraisemblable de délabrement, de misère, de violence, avec une séparation totalement hermétique entre le monde blanc, prospère et privilégié de l'université et les quartiers noirs à l'abandon tout autour (le campus de Hyde Park est bordé sur trois côtés par le ghetto du South Side et sur le quatrième par le lac Michigan). Ça me questionnait profondément au quotidien.

canaque en Nouvelle-Calédonie » (avec J.M. Kohler et P. Pillon), Cahiers ORSTOMSérie sciences humaines, 21-2/3, 1985, p. 203-228 [http://horizon.documentation.ird.fr/exldoc/pleins textes/pleins textes_4/sci hum/36869.pdf] ; "Communautés canaques et société coloniale: notes complémentaires sur la "question canaque" », Actes de la recherche en sciences sociales, 61, mars 1986, p. 56-64; «The Dark Side of the Classroom in New Caledonia: Ethnic and Class Segregation in Nouméa's Primary School System », Comparative Education Review, 33-2, mai 1989, p. 194-212.

${ }^{7}$ Loïc Wacquant, L'École inégale. Éléments pour une sociologie de l'enseignement en Nouvelle-Calédonie, Nouméa et Paris, Éditions de l'ORSTOM et Institut Culturel Mélanésien, 1985, 212 p. 


\section{Labyrinthe, $n^{\circ} 31$}

C'est alors qu'intervient la deuxième rencontre décisive de ma vie intellectuelle, celle avec William Julius Wilson.

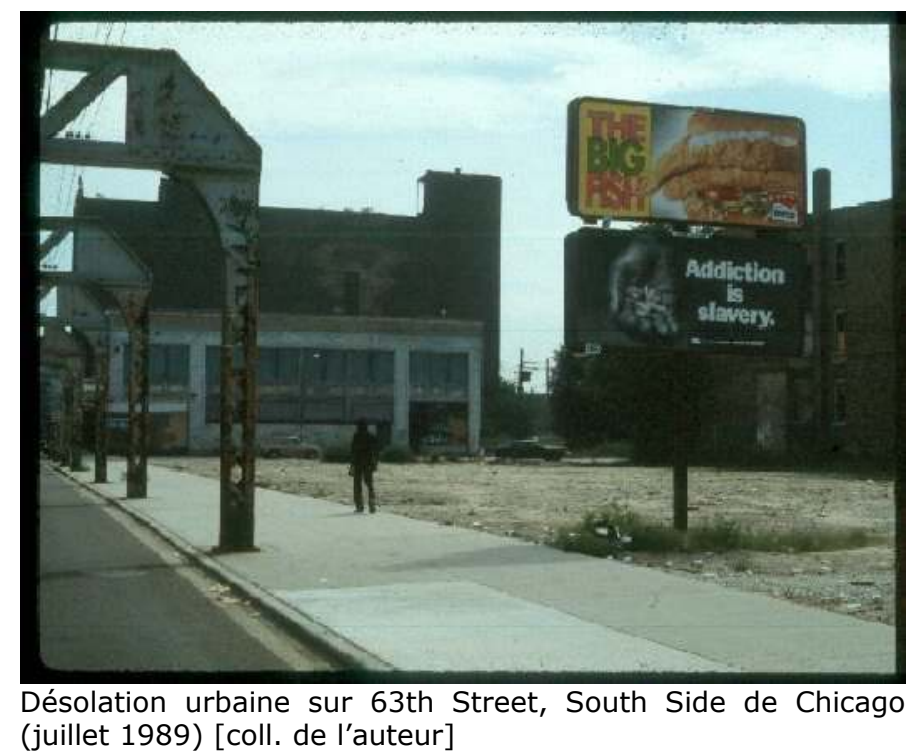

Wilson est le plus éminent sociologue noir américain de la seconde moitié du $\mathrm{XX}^{\mathrm{e}}$ siècle et le grand spécialiste de la question des rapports entre « race et classe » dans ce pays. Il m'a proposé de travailler avec lui à un projet sur la pauvreté urbaine (en gros, le programme de recherche tracé par son livre The Truly Disadvantaged) et je suis rapidement devenu son collaborateur proche et co-auteur ${ }^{8}$. J'ai donc eu la chance d'aller tout de suite au cœur du sujet, mais aussi de voir de près comment fonctionnait ce débat scientifique et politique au plus haut niveau, notamment dans les instituts philanthropiques et les think tanks. C'est ainsi que j'ai entamé mes investigations, d'abord avec Wilson puis par moi-même, sur la transformation du ghetto noir après les années 1960, en essayant de sortir de la vision pathologisante qui

\footnotetext{
${ }^{8}$ William Julius Wilson, The Truly Disadvantaged: The Inner City, the Underclass, and Public Policy, Chicago, University of Chicago Press, 1987. L'ouvrage fait suite à The Declining Significance of Race: Blacks and Changing American Institutions, Chicago, University of Chicago Press, 1978.
} 


\section{Le corps, le ghetto et l'État pénal}

imprègne et biaise les travaux sur la question'. J'ai une grande dette envers Bill Wilson, qui a été un mentor à la fois exigeant et généreux : il m'a stimulé et soutenu, mais aussi donné la liberté de diverger d'avec ses analyses, parfois de manière frontale.

L'ethnographie a joué alors un rôle-pivot, pour deux raisons. D'un côté, j'ai suivi plus de cours en anthropologie qu'en sociologie, parce que le département de sociologie de Chicago était très terne intellectuellement et parce que j'étais viscéralement attaché à une conception unitaire de la science sociale héritée de ma formation française. Les travaux et les encouragements au travail de John et Jean Comaroff, de Marshall Sahlins, de Bernard Cohn et Raymond Smith m'ont poussé dans la direction du travail de terrain. De l'autre, j'ai voulu très vite trouver un point d'observation directe à l'intérieur $\mathrm{du}$ ghetto, parce que la littérature existante sur le sujet était le produit d'un «regard lointain» qui me semblait foncièrement biaisé sinon aveugle. Cette littérature est dominée par l'approche statistique, déployée de très haut, par des chercheurs qui le plus souvent n'ont aucune connaissance première, voire même secondaire, de ce qui fait la réalité ordinaire des quartiers déshérités de la Ceinture noire, et qui remplissent ce vide avec des stéréotypes tirés du sens commun ordinaire, journalistique ou universitaire. J'ai voulu reconstruire la question du ghetto à partir d'en bas, sur la base d'une observation précise de la vie quotidienne des habitants de cette terra non grata mais aussi, pour cette raison même, incognita ${ }^{10}$.

Susana Durão - C'est cette sociologie " au ras du sol » vous a amené à fréquenter les rings de boxe?

Loïc Wacquant - Je jugeais impossible, épistémologiquement et moralement, de travailler sur le ghetto sans le connaître de première main puisqu'il était là, sur le pas de ma porte (l'été, on entendait clairement les coups de feu qui claquaient la nuit de l'autre côté de la rue) et que les travaux établis me semblaient pleins de notions

\footnotetext{
${ }^{9}$ Loïc Wacquant, «Three Pernicious Premises in the Study of the American Ghetto », International Journal of Urban and Regional Research, 21-2, juin 1997, p. 341-353 [trad. française : «Trois tendances pernicieuses dans l'étude du ghetto américain », $M$ : Mensuel Marxisme Mouvement, numéro spécial "Mégalopole USA», 85-86, novembre-décembre 1996, p. 36-41].

${ }^{10}$ Loïc Wacquant, "“The Zone": Le métier de "hustler" dans le ghetto noir américain ", Actes de la recherche en sciences sociales, 93, juin 1992, p. 38-58.
} 


\section{Labyrinthe, $n^{\circ} 31$}

académiques improbables ou pernicieuses, comme le mythe savant de l'underclass qui avait alors le vent en poupe ${ }^{11}$. Après quelques tentatives avortées, j'ai trouvé par accident une salle de boxe dans Woodlawn, à trois pâtés de maisons de mon appartement, et je m'y suis inscrit en disant que je souhaitais apprendre à boxer, tout simplement parce qu'il n'y avait rien d'autre à faire dans le contexte. En fait, je n'avais absolument aucune curiosité ni intérêt pour le monde pugilistique en soi. La salle devait juste être un point d'observation dans le ghetto, un lieu de rencontre avec des informateurs potentiels.

Mais très vite, le gym s'est révélé être non seulement une très belle fenêtre sur la vie quotidienne des jeunes du quartier mais aussi un microcosme complexe, avec une histoire et une culture, une vie sociale, esthétique, émotionnelle et morale à lui très intenses et très riches. Je me suis lié d'une amitié très forte, charnelle, avec les habitués de la salle et avec le vieux coach, DeeDee Armour, qui est devenu une sorte de père adoptif ${ }^{12}$. Graduellement, je me suis trouvé aimanté par le magnétisme de la "Sweet Science », au point de passer le plus clair de mon temps à la salle. Au bout d'un an s'est imposée à moi l'idée de creuser un deuxième sujet: la logique sociale d'un métier du corps. Qu'est-ce qui fait vibrer les boxeurs, pourquoi s'engagent-ils dans ce métier si dur et destructeur entre tous, comment acquièrent-ils l'envie et les habiletés nécessaires pour durer? Quels sont les rôles de la salle, de la rue, de la violence environnante et du mépris racial, de l'intérêt et du plaisir, mais encore, dans tout ça, de la croyance collective dans la transcendance personnelle? Comment crée-t-on une compétence sociale qui est une compétence incorporée, qui se transmet par une pédagogie silencieuse des organismes en action? Bref, comment se fabrique et se déploie l'habitus pugilistique ${ }^{13}$ ?

\footnotetext{
${ }^{11}$ Loïc Wacquant, «L' "underclass" urbaine dans l'imaginaire social et scientifique américain », dans Serge Paugam (dir.), L'Exclusion : l'état des savoirs, Paris, Éditions La Découverte, 1996, p. 248-262.

12 Loïc Wacquant, «Chicago Fade: remettre le corps du chercheur en scène », Quasimodo, 7, printemps 2002, p. 171-179.

${ }^{13}$ Loïc Wacquant, « Corps et âme : notes ethnographiques d'un apprenti-boxeur », Actes de la recherche en sciences sociales, 80, novembre 1989, p. 33-67; «Protection, discipline et honneur: une salle de boxe dans le ghetto américain ", Sociologie et sociétés, 27-1, printemps 1995, p. 75-89; «The Pugilistic Point of View : How Boxers Think and Feel About Their Trade », Theory \& Society, 24-4, août 1995, p. 489-535;
} 


\section{Le corps, le ghetto et l'État pénal}

C'est comme ça que je me suis retrouvé à mener de front deux projets connexes, très différents mais en fait étroitement liés : une microsociologie charnelle de l'apprentissage de la boxe comme métier du corps sous-prolétarien dans le ghetto, donnant de cet univers une «coupe » particulière vu d'en bas et de l'intérieur; et une macrosociologie historique et théorique $d u$ ghetto comme instrument de fermeture raciale et de domination sociale, offrant une perspective généralisante tirant vers l'extérieur et vers le haut.

Susana Durão - C'est au moment où vous conduisez votre travail de terrain sur le South Side qu'explose le discours-panique sur la "ghettoïsation » des banlieues populaires en France.

Loïc Wacquant - Précisément. En 1990, après les émeutes de Vauxen-Velin, se cristallise en France - puis dans les autres pays européens - une «panique morale» autour des quartiers périphériques déstabilisés par la désindustrialisation et le chômage de masse, dont on dit soudain qu'ils se métamorphosent en ghetto à l'américaine, avec les immigrés dans le rôle des Noirs en quelque sorte. Or, j'étais à Chicago plongé dans mon enquête au sein du South Side, et cette légende médiatique, rapidement partagée par les politiques et par certains chercheurs (pas toujours les mieux informés !), me paraissait proprement ubuesque. Là encore on nageait dans les stéréotypes et les clichés, fondés en l'occurrence sur une double ignorance : l'ignorance de ce qu'est la banlieue populaire française à l'ère postfordiste et l'ignorance du ghetto noir américain. Le produit de ces deux ignorances cumulées était un discours en décalage complet par rapport à la réalité, mais qui a d'emblée exercé un puissant effet de « prophétie auto-réalisante », parce qu'il était repris par tous et partout et qu'il a très vite guidé les politiques publiques - et notamment la politique dite de la ville, avec l'annonce périodique de «Lois antighetto » aussi hypocrites qu'inefficaces.

J'ai jugé que j'avais un devoir à la fois scientifique et civique d'intervenir dans ce (faux) débat pour en récuser les termes par l'étude méthodique des transformations des quartiers de relégation, ces

«Pugs at Work : Bodily Capital and Bodily Labor Among Professional Boxers », Body \& Society, 1-1, mars 1995, p. 65-94 [trad. française : « La fabrique de la cogne : capital corporel et travail du corps chez les boxeurs professionnels », Quasimodo, 7, Spring 2003, pp. 181-201,]; «The Prizefighter's Three Bodies», Ethnos: Journal of Anthropology, 63-3, novembre 1998, p. 325-352. 


\section{Labyrinthe, $n^{\circ} 31$}

espaces stigmatisés dans lesquels sont repoussées les populations marginalisées à la fois sous l'angle matériel et sous l'angle de l'honneur sur les deux rives de l'Atlantique. J'ai donc entamé une comparaison, point par point, entre l'évolution du ghetto noir américain après les grandes révoltes des années 1960 et l'évolution des banlieues populaires en France après le milieu des années 1970, soit durant la phase de désindustrialisation, qui a d'abord donné lieu a une série d'articles principalement orientés vers le débat européen ${ }^{14}$. Pour comparer le South Side de Chicago avec la banlieue parisienne, j'ai fait une enquête de terrain en 1989-91 dans la cité des Quatre mille, dans la ville de La Courneuve au nord-est de Paris, et dans les couloirs des administrations qui mettaient en œuvre la soi-disant politique de la ville. À son terme, ce travail débouche sur une triple clarification, empirique, théorique et politique : je retrace comment le "ghetto communautaire» du milieu du $\mathrm{XX}^{\mathrm{e}}$ siècle s'est mué en « hyperghetto » du côté américain; comment les territoires ouvriers de la périphérie urbaine européenne sont entrés en décomposition, mais en s'éloignant du schéma du ghetto, contrairement au discours dominant, au point qu'on peut les caractériser comme des antighettos; et je démontre que c'est l'État qui est le déterminant majeur des formes que prend la marginalité urbaine sur les deux continents ${ }^{15}$.

\footnotetext{
${ }^{14}$ Loïc Wacquant, « Banlieues françaises et ghetto noir américain : de l'amalgame à la comparaison », French Politics and Society, 10-4, automne 1992, p. 81-103; « Pour en finir avec le mythe des "cités-ghettos": les différences entre la France et les ÉtatsUnis ", Annales de la recherche urbaine, 52, septembre 1992, p. 20-30 [http://www2.urbanisme.equipement.gouv.fr/cdu/datas/annales/wacquan.htm, page consultée le 30 août 2008] ; «Décivilisation et démonisation : la mutation du ghetto noir américain », dans Christine Fauré et Tom Bishop (dir.), L'Amérique des français, Paris, Éditions François Bourin, 1992, p. 103-125 ; «Urban Outcasts : Stigma and Division in the Black American Ghetto and the French Urban Periphery », International Journal of Urban and Regional Research, 17-3, septembre 1993, p. 366-383.

${ }^{15}$ Loïc Wacquant, «Ghetto, banlieues, État : réaffirmer la primauté du politique», Nouveaux regards, 33, avril-juin 2006, p. 62-66; "Ghettos, Anti-Ghettos: An Anatomy of the New Urban Poverty ", Thesis Eleven, août 2008, p. 113-118.
} 


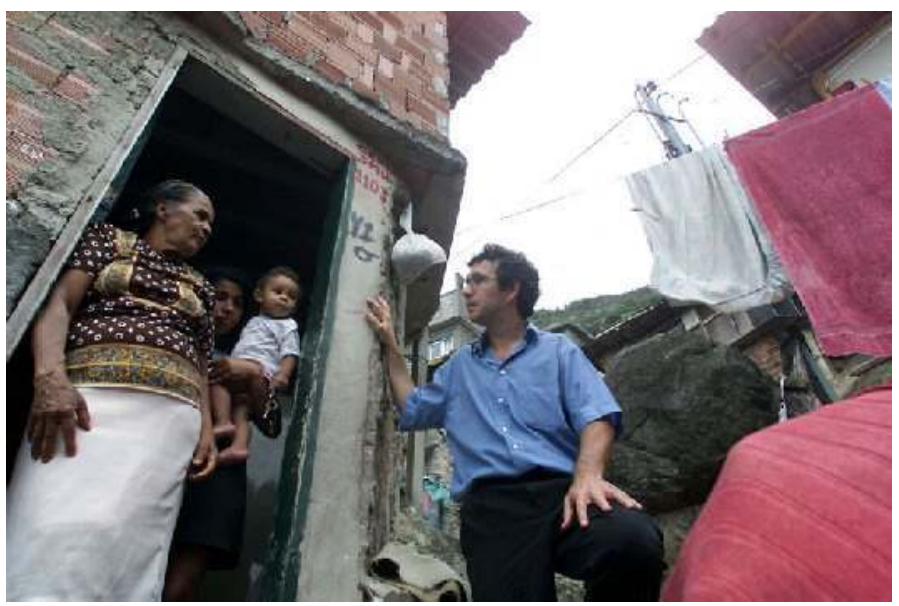

Dans la favela de Santa Marta, Rio de Janeiro (avril 2001) [coll. de l'auteur]

Pendant que je menais mon travail sur la boxe et le ghetto, j'étais en contact permanent avec Pierre Bourdieu, qui m'a constamment encouragé. Il est venu plusieurs fois à Chicago, où il a visité le gym et rencontré DeeDee et mes amis boxeurs. C'est lors de ces visites que nous avons élaboré le projet d'un livre qui expliciterait le cœur théorique de son travail, visant à l'origine un public anglo-américain, puisque c'est sur ce front que les distorsions et les obstacles à une appropriation fructueuse de ses modèles étaient les plus forts. Nous avons consacré trois ans à la rédaction de ce livre, intitulé $A n$ Invitation to Reflexive Sociology ${ }^{16}$, que nous avons écrit directement en anglais, et qui a très vite été traduit en français puis dans une vingtaine de langues. Sociologie du ghetto, ethnographie du corps habile, comparaison transatlantique et travail théorique avec Bourdieu : tout s'est construit ensemble et en même temps, et tout se tient.

\footnotetext{
${ }^{16}$ Loïc Wacquant et Pierre Bourdieu, An Invitation to Reflexive Sociology, Chicago, University of Chicago Press et Cambridge [UK, Polity Press], 1992 [trad. française abrégée : Réponses. Pour une anthropologie réflexive, Paris, Seuil, 1992].
} 


\section{LE ROC DE L'ÉTAT PÉNAL}

Susana Durão - Mais alors comment surgissent les prisons dans ce programme d'études?

Loïc Wacquant - Là encore, comme pour l'anthropologie du pugilisme, c'était totalement imprévu : c'est la logique de la recherche et les surprises du terrain qui m'ont forcé à «entrer en prison» - au sens figuré s'entend. En dressant l'histoire de la vie de mes amis boxeurs à la salle de Woodlawn, je me suis aperçu que tous presque tous avaient fait de la détention. J'ai alors réalisé que la prison est une institution à la fois centrale et banale dans l'horizon des organisations auxquelles les jeunes du ghetto ont affaire et qui les font trébucher comme un gros roc dans leur jardin personnel, qu'on ne peut ni enlever ni contourner, et qui change tout au paysage social.

Par exemple, mon ami et partenaire de ring Ashante avait fait six ans de prison au sortir de l'adolescence; il avait d'ailleurs appris à boxer derrière les barreaux. À sa sortie du pénitencier, il a trouvé refuge à la salle, qui l'a protégé de la rue, et il a poursuivi une carrière de boxeur. Puis, quand sa carrière sur le ring a capoté et que le gym a fermé, il est retombé dans l'économie illégale et il s'est retrouvé à nouveau sous les verrous plusieurs fois. J'allais périodiquement le sortir de taule en payant sa caution et son avocat... Voir son meilleur ami jeté en prison au sortir du tribunal, ça vous secoue existentiellement et intellectuellement ! C'est cette expérience qui m'a conduit à faire une enquête de terrain pilote dans les maisons d'arrêt états-uniennes en 1998-99, à Los Angeles puis Chicago et New York (avec des incursions au Brésil), pour comprendre ce qui lui arrivait ${ }^{17}$. Le but, là encore, était de se donner les moyens de percer l'écran des discours dominants sur la prison et des analyses distantes et mécaniques de la criminologie, qui néglige la texture des rapports carcéraux au quotidien : l'emprisonnement, c'est avant tout des corps contraints, et tout ce que cela imprime au niveau des catégories, des désirs, du sens de soi et des relations aux autres.

De fait, on ne peut pas comprendre la trajectoire du sousprolétariat noir américain après les émeutes qui ont secoué le ghetto

\footnotetext{
${ }^{17}$ Loïc Wacquant, «The Curious Eclipse of Prison Ethnography in the Age of Mass Incarceration », Ethnography, 3-4, Winter 2002, n. spécial « In and Out of the Belly of the Beast », p. 371-397.
} 


\section{Le corps, le ghetto et l'État pénal}

dans les années 1970 sans prendre dans son collimateur analytique l'expansion stupéfiante de l'État pénal lors des trois dernières décennies du siècle. Entre 1975 et 2000, les États-Unis ont multiplié par cinq leur population sous écrou pour devenir le leader mondial de l'incarcération avec 2 millions de détenus - chose que j'ignorais alors et dont je ne tenais aucun compte analytique, comme tout sociologue travaillant sur race et classe en Amérique (le premier qui l'a fait est un juriste, Michael Tonry, dans Malign Neglect, un livre-clef paru en 1995, qui a attiré mon attention parce que je voulais utiliser ce titre pour un de mes ouvrages ${ }^{18}$ ).

Comment s'explique cette hyperinflation carcérale ? La première réponse, celle de l'idéologie dominante et de la recherche officielle, est de dire qu'elle est liée au crime. Mais la courbe de la criminalité a stagné de 1973 à 1993 avant de chuter fortement, au moment même où l'emprisonnement s'envolait. Deuxième mystère: alors que la proportion des Noirs dans chaque « cohorte » de criminels est allée en diminuant pendant vingt ans, leur part de la population carcérale, elle, n'a cessé d'augmenter. Pour résoudre ces deux énigmes, il faut sortir du schéma «crime et châtiment» et repenser la prison comme une institution politique, une composante centrale de l'État. Et l'on découvre alors que le surgissement de l'État pénal est le résultat d'une polique de pénalisation de la misère qui répond à la montée de l'insécurité salariale et de l'effondrement du ghetto comme mécanisme de contrôle d'une population doublement marginalisée au double plan matériel et symbolique ${ }^{19}$.

Susana Durão - Et, comme vous le montrez dans Les Prisons de la misère (1999), l'expansion de l'État pénal aux États-Unis est elle-

\footnotetext{
${ }^{18}$ Michael Tonry, Malign Neglect: Race and Punishment in America, New York, Oxford University Press, 1995.

${ }^{19}$ Loïc Wacquant, «Crime et Châtiment en Amérique de Nixon à Clinton », Archives de politique criminelle, 20, printemps 1998, p. 123-138; 2000, «The New "Peculiar Institution": On the Prison as Surrogate Ghetto », Theoretical Criminology, 4-3, no. spécial sur « New Social Studies of the Prison », p. 377-389 [trad. française, « La prison comme substitut du ghetto: la nouvelle "institution particulière" de l'Amérique », Philosophie, Critique, Littérature, 24, Octobre 2000, p. 17-33] ; «Deadly Symbiosis : When Ghetto and Prison Meet and Mesh », Punishment \& Society, 3-1 hiver 2001, p. 95-133 [trad. française abrégée: «Symbiose fatale: quand ghetto et prison se ressemblent et s'assemblent», Actes de la recherche en sciences sociales, 139, Septembre 2001, p. 31-52].
} 
même liée à l'atrophie de l'État social... ${ }^{20}$

Loïc Wacquant - Au moment où je me plongeais dans les statistiques pénitentiaires pour décrypter la stupéfiante ascension de l'État pénal en Amérique, Clinton avalisait la «welfare reform» de 1996, concoctée par la faction la plus réactionnaire du parti républicain. L'abolition du droit à l'assistance sociale pour les femmes démunies et son remplacement par l'obligation du salariat forcé (dit worfare) est un scandale historique, la mesure la plus régressive prise par un président supposé progressiste durant tout le $\mathrm{XX}^{\mathrm{e}}$ siècle. Par indignation politique, j'ai écrit un papier dans Le Monde diplomatique, puis un article plus fouillé pour une revue de géographique politique, Hérodote ${ }^{21}$. En analysant les implications de cette réforme, j'ai réalisé que l'atrophie organisée du secteur social et l'hypertrophie du secteur pénal de l'État américain étaient non seulement concomittantes et complémentaires, mais encore qu'elles visaient la même population stigmatisée à la marge du salariat. Il devenait clair que la «main invisible » du marché dérégulé appelle et nécessite le renforcement du «poing de fer» de la justice criminelle en bas de la structure des classes.

C'est ce que j'ai essayé de montrer dans Les Prisons de la misère, en suivant la diffusion internationale de la politique de «tolérance zéro » qui est le fer de lance de pénalisation de la pauvreté. Ce livre a été très vite traduit en trois, six, douze langues, parce que cette politique de «contention punitive» des couches précarisées du nouveau prolétariat urbain s'est répandue à travers la terre entière, dans le sillage du néolibéralisme économique. C'est ainsi que je me suis détourné momentanément du ghetto, poussé par l'urgence politique et presque contre mon gré, pour analyser plus avant les transformations des politiques pénales dans leurs rapports avec les politiques sociales.

\footnotetext{
${ }^{20}$ Loïc Wacquant, Les Prisons de la Misère, Paris, Éditions du Seuil, 1999.

${ }^{21}$ Loïc Wacquant, «Les pauvres en pâture: la nouvelle politique de la misère en Amérique », Hérodote, 85, printemps 1997, p. 21-331; 1996, «De l'État charitable à l'État pénal : notes sur le traitement politique de la misère en Amérique », Regards sociologiques, 11, p. 30-38; voir également le numéro des Actes de la recherche en sciences sociales, 124, septembre 1998, consacré au basculement « De l'État social à l'État pénal », avec des articles de David Garland, Katherine Beckett et Bruce Western, Dario Melossi et Loïc Wacquant.
} 


\section{Le corps, le ghetto et l'État pénal}

Susana Durão - Mais l'analyse du rôle de la prison vous ramène aux quartiers de relégation puisque ce sont eux qui sont la cible privilégiée du déploiement de l'État pénal.

Loïc Wacquant - En fait, sans le planifier, j'ai écrit une sorte de trilogie sur les rapports entre pauvreté/ethnicité, État social et État pénal à l'ère du néolibéralisme triomphant, mais publiée dans le désordre. Le premier volume, c'est Parias urbains, où, ayant réfuté la thèse de la convergence transatlantique des formes de marginalité urbaine, je formule le diagnostic de l'émergence d'un nouveau régime de pauvreté urbaine, différent du régime "fordiste-keynésien » qui prévalait jusque dans les années $1970^{22}$. Je l'appelle marginalité avancée parce qu'elle est n'est ni résiduelle ni cyclique, mais devant nous, inscrite dans le devenir des sociétés avancées soumises aux torsions de la dérégulation capitaliste ${ }^{23}$. Pour aller vite, la marginalité avancée, qui supplante le ghetto côté américain et le territoire ouvrier traditionnel côté européen, est le produit de la fragmentation du salariat, de la disconnection fonctionnelle entre les quartiers de relégation et l'économie nationale et mondiale, de la stigmatisation territoriale et de la rétraction des protections assurées par l'État social.

Comment l'État va-t-il réagir à la montée de cette marginalité et gérer le cortège de "problèmes sociaux » qu'elle charrie : chômage, sans-abrisme, criminalité, drogues, jeunesse désœuvrée et enragée, exclusion scolaire, dissolution familiale et sociale, etc. Comment contenir ses réverbérations et, en même temps, inciter les couches précaires du nouveau prolétariat urbain, ce qu'on peut appeler le "précariat», à accepter les emplois instables et sous-payés de l'économie dérégulée des services? La réponse est donnée dans le deuxième volume: Punishing the Poor dissèque l'invention d'un «nouveau gouvernement de l'insécurité sociale» qui allie la discipline du workfare et la contrainte d'un appareil policier et pénal surdimensionné et hyperactif ${ }^{24}$. En 1971, Frances Fox Piven et

\footnotetext{
${ }^{22}$ Loïc Wacquant, Parias urbains. Ghetto, banlieues, État, Paris, La Découverte, 2006.

${ }^{23}$ Loïc Wacquant, «Les banlieues populaires à l'heure de la marginalité avancée », Sciences humaines, 4, automne 1996, p. 30-33.

${ }^{24}$ Loïc Wacquant, Punishing the Poor: The New Government of Social Insecurity, Durham and London, Duke University Press, 2008. Note: cet ouvrage est la version complète et conforme d'un livre dont une version contrefaisante, truquée et tronquée, a été publiée illégalement par Agone en 2004 sous le titre Punir les pauvres et que l'auteur ne reconnaît pas comme son travail.
} 


\section{Labyrinthe, $n^{\circ} 31$}

Richard Cloward ont publié un livre audacieux, devenu depuis un classique de la science sociale, intitulé Regulating the Poor ${ }^{25}$. Ils y montrent que les politiques sociales, et notamment l'assistance aux pauvres, évoluent de manière cyclique, par contraction et expansion, de sorte à pousser les démunis sur le marché du travail en période économique faste et à empêcher qu'ils ne se révoltent en période creuse. Ma thèse, c'est que, trente ans plus tard, cette « régulation des pauvres » ne passe plus par le seul welfare mais implique une chaîne institutionnelle qui lie entre eux les secteurs assistanciel et pénitentiel de l'État. Ce qui implique que si on veut comprendre les politiques de gestion des populations à problèmes en bas de la structure des classes et des places, il faut étudier ensemble ce que Bourdieu appelle la « main gauche » et la «main droite » de l'État. La politique sociale et la politique pénale convergent et fusionnent: la même philosophie behavioriste, les mêmes notions de responsabilité individuelle et de contrat, les mêmes dispositifs de surveillance et de fichage, les mêmes techniques de supervision, de «dégradation rituelle» (au sens de Garfinkel $^{26}$ ) et de sanction des écarts de comportement informent l'action des services sociaux, transformés en tremplin vers l'emploi précaire, et de la police, la justice et la prison, à qui on demande de juguler les populations marginalisées.

Vient alors le troisième volume, qui est celui que j'ai écrit en premier pour des raisons d'urgence politique, Les Prisons de la Misère, qui démontre les causes et démonte les mécanismes de l'internationalisation de la pénalisation de la marginalité urbaine, avec la diffusion de la stratégie policière de «tolérance zéro » à l'échelle planétaire, concommittante de la diffusion des politiques économiques néolibérales ${ }^{27}$. S'y ajoute un quatrième volume, Deadly Symbiosis, qui montre comment la division ethnoraciale lubrifie l'expansion de l'État pénal et accélère la transition de la gestion sociale vers la gestion punitive de la pauvreté, et comment en retour, par son action

\footnotetext{
${ }^{25}$ Frances Fox Piven \& Richard Cloward, Regulating the Poor: The Functions of Public Welfare, New York, Pantheon Books (1971), éd. augmentée 1994.

${ }^{26}$ Harold Garfinkel, "Conditions of Successful Degradation Ceremonies », American Journal of Sociology, 61, septembre 1956, p. 240-244.

${ }^{27}$ Loïc Wacquant, «The Penalisation of Poverty and the Rise of Neoliberalism », European Journal on Criminal Policy and Research, 9-4, hiver 2001, p. 401-412; «Towards a Dictatorship over the Poor? Notes on the Penalization of Poverty in Brazil », Punishment \& Society, 5-2, avril 2003, p. 197-205.
} 
matérielle et symbolique, l'institution carcérale redéfinit et redéploie le stigmate ethnique ou ethnonationa ${ }^{28}$. Ce livre mêle ethnographie, histoire sociale, théorie sociologique et philosophie juridique, et teste le modèle de la fusion structurale et fonctionnelle des quartiers de relégation et du système carcéral, construit sur le cas des États-Unis, en le transportant en Europe pour expliquer le suremprisonnement des immigrés postcoloniaux, et au Brésil pour y rendre compte de la «militarisation» des clivages urbains dans la ville duelle.

Susana Durão - Il existe donc non seulement un fil conducteur existentiel mais aussi une couture théorique qui relie entre elles ces thématiques très différentes.

Loïc Wacquant - Ce sont des objets empiriques qui sont en apparence très dispersés et traditionnellement traités par des secteurs distincts de la recherche qui ne communiquent pas entre eux: l'anthropologie du corps, la sociologie de la pauvreté et de la domination raciale, et la criminologie. Les gens qui travaillent sur le corps, la culture quotidienne, la production du désir, ne s'intéressent généralement pas à l'État; ceux qui décryptent les politiques de justice, de manière typique, ne se préoccupent guère de marginalité urbaine ou de politique sociale; et les pénologues ne prêtent attention ni au corps ni aux politiques de l'État qui ne concernent pas officiellement la lutte contre le crime. Mon argument, c'est qu'on ne peut pas séparer le corps, l'État social ou pénal et la marginalité urbaine : il faut les saisir et les expliquer ensemble, dans leurs imbrications mutuelles. Et la rampe de lancement théorique de cette ogive analytique à trois têtes, c'est l'Invitation to Reflexive Sociology, qui contient tous les concepts-clefs et les principes méthodologiques mis en œuvre dans les autres livres.

\footnotetext{
${ }^{28}$ Loïc Wacquant, «Race as Civic Felony », International Social Science Journal, 181, printemps 2005, p. 127-142 [trad. française : "La race comme crime civique", idem, 183, mars 2005] ; et Deadly Symbiosis: Race and the Rise of the Penal State, Cambridge, Polity Press, 2009.
} 


\section{L'ETHNOGRAPHIE COMME INSTRUMENT DE RUPTURE ET DE CONSTRUCTION}

Susana Durão - La place centrale de l'ethnographie dans votre parcours intellectuel est claire, mais j'aimerais que vous précisiez le rôle qu'elle a joué dans les différentes recherches que vous avez réalisées, car on n'associe pas couramment le nom de Bourdieu à l'ethnographie.

Loïc Wacquant - C'est bien à tort, car, comme je l'ai montré dans l'article qui ouvre le numéro spécial d'Ethnography sur «Pierre Bourdieu in the Field», Bourdieu était l'un des praticiens les plus originaux de cette approche, qui de plus a été décisive dans la gestation de son projet scientifique ${ }^{29}$. Il a écrit non seulement des textes qui sont des joyaux de l'art ethnographique, comme «Le sens de l'honneur » (1965) et «La maison kabyle ou le monde renversé » (1971), mais encore l'observation de terrain joue un rôle-pivot dans tous ses livres majeurs, des Héritiers aux Règles de l'art en passant par La Distinction ${ }^{30}$.

Pour ne prendre que son travail de jeunesse, Bourdieu nous a légué une extraordinaire ethnographie comparée, menée des deux côtés de la Méditerranée, des transformations cataclysmiques des structures sociales et mentales des sociétés paysannes, en Kabylie sous l'effet de la pénétration coloniale française et de la guerre de libération nationale, et dans son village du Béarn sous l'effet de la généralisation de la scolarisation, l'ouverture de l'espace villageois aux échanges marchands et l'influence de la culture urbaine par le biais des médias $^{31}$. Ceux qui persistent à faire de lui un «théoricien de la reproduction » seraient bien avisés de relire ces études... Bourdieu faisait de l'ethnographie comparée, menée de front sur plusieurs sites

\footnotetext{
${ }^{29}$ Loïc Wacquant, «Following Pierre Bourdieu into the Field», Ethnography, 5-4, décembre 2004, p. 387-414.

${ }^{30}$ Pierre Bourdieu, «Sens de l'honneur» et «Maison Kabyle» dans Esquisse d'une théorie de la pratique. Précédée de trois études d'ethnologie kabyle [Genève, Éditions Droz, 1972], rééd. Paris, Seuil/Points, 2000.

${ }^{31}$ Pierre Bourdieu (avec A. Darbel, J.-P. Rivet, C. Seibel), Travail et travailleurs en Algérie, Paris-La Haye, Mouton, 1963 ; Pierre Bourdieu et Abdelmalek Sayad, Le Déracinement. La crise de l'agriculture traditionnelle en Algérie, Paris, Éd. de Minuit, 1964 ; Pierre Bourdieu, Le Bal des célibataires. Crise de la société paysanne en Béarn, Paris, Seuil, [1962], 2002.
} 


\section{Le corps, le ghetto et l'État pénal}

et combinée avec l'analyse statistique, trente ans avec que ne survienne la mode de l'ethnographie «multi-site»- qui souvent cache mal une pratique qui s'apparente plus au tourisme culturel qu'à un travail de terrain digne du nom. Et une ethnographie qui, loin de céder à l'exotisme et à l'empiricisme, était fermement guidée par un projet théorique qu'elle venait en retour alimenter: la plupart des concepts-clefs de Bourdieu, comme celui d'habitus, ont leur origine dans un puzzle rencontré sur le terrain. De plus, il y a toujours eu dans le sillage de Bourdieu, au Centre de sociologie européenne et ailleurs, des praticiens de première force de l'ethnographie: je pense notamment à Abdelmalek Sayad, à Stéphane Beaud et Michel Pialoux, à Yvette Delsaut ou encore à Monique et Michel Pinçon ${ }^{32}$.

C'est dire que je n'aurais pas manqué de modèles à imiter si j'avais voulu devenir ethnographe par une sorte de décision délibérée. Mais la question de faire ou non du terrain ne s'est jamais posée en termes de vocation méthodologique pour moi. C'est plutôt la méthode qui est venue à moi comme la plus adéquate pour résoudre le problème concret auquel j'étais confronté, qui, à Chicago, n'était pas seulement de me «rapprocher» du ghetto pour en acquérir une connaissance pratique et ressentie de l'intérieur, mais aussi de me doter d'un instrument de déconstruction des catégories à travers lesquelles la «Ceinture noire » américaine était alors perçue et pensée dans le débat universitaire et politique. Mon intention initiale était de m'appuyer sur une ethnographie de la scène urbaine du South Side pour percer le double écran que formaient le discours préfabriqué sur le ghetto comme lieu de désorganisation sociale - espace de violence, de déviance, de vide, caractérisé par l'absence ou le manque - qui découle du point de vue externe et exotisant qu'adopte la sociologie établie, et la fable académique de l'underclass, cette catégorieépouvantail apparue dans les années 1980-90 dans l'imaginaire social et scientifique des États-Unis pour expliquer de manière parfaitement tautologique l'effondrement du ghetto noir par le «comportement

\footnotetext{
${ }^{32}$ Abdelmalek Sayad, Un Nanterre algérien, terre de bidonvilles, Paris, Éditions Autrement, 1995 ; Stéphane Beaud et Michel Pialoux, Retour sur la condition ouvrière. Enquête aux usines Peugeot de Sochaux-Montbéliard, Paris, Fayard, 1999 ; Yvette Delsaut, La Place du maître. Une chronique des écoles normales d'instituteurs, Paris, L'Harmattan, 1992 ; Michel Pinçon et Monique Pinçon-Charlot, Voyage en grande bourgeoisie. Journal d'enquête, Paris, Presses Universitaires de France, 1997.
} 
antisocial $\gg$ de ses membres ${ }^{33}$.

L'observation ethnographique m'a permis d'effectuer une double rupture, avec la représentation médiatico-politique dominante et avec le sens commun savant de l'époque, lui-même fortement contaminé par la doxa nationale. Même chose du côté français où la confrontation entre ce que $\mathrm{j}$ 'ai vu et entendu dans les services du Ministère de la ville et dans la cité des Quatre mille à La Courneuve m'a permis de passer au crible les préconceptions bureaucratiques et demi-savantes qui font obstacle à la construction de l'objet «banlieues ».

Susana Durão - Cette intention est explicitée dans le prologue méthodologique de Parias urbains où vous mentionnez l'apport de l'ethnographie parmi cinq principes pour guider la sociologie comparée de la marginalité urbaine.

Loïc Wacquant - Parias Urbains n'est pas une monographie de terrain au sens classique, puisque l'analyse articule les niveaux « micro » du quartier, «méso » de la ville et du cadre politique local, et «macro » de l'économie et l'État national, et combine observation directe, données statistiques et mise en perspective historique et comparative. $^{34}$ Mais l'ethnographie n'en remplit pas moins une fonction majeure dans deux registres analytiques : comme instrument de rupture avec la doxa politique et intellectuelle, comme je viens de l'indiquer, et comme outil de construction théorique.

Les observations consignées au jour le jour dans le ghetto noir de Chicago dans le sillage de mes collègues de la salle de boxe sur leurs rapports avec les employeurs, les agences de l'aide sociale, la police, les gangs, l'école, etc., m'ont permis d'élaborer les notions idéalestypiques que je déploie pour décrypter les pratiques sociales et de l'expérience vécue de la pauvreté dans le cœur ségrégué de la métropole étasunienne. Ainsi la notion d'hyperghetto exprime

\footnotetext{
${ }^{33}$ Loïc Wacquant, «Three Pernicious Premises in the Study of the American Ghetto », International Journal of Urban and Regional Research, 21-2, June 1997, «Events and Debate », p. 341-353 (et les 8 réponses dans les numéros suivants) [trad. française : "Trois tendances pernicieuses dans l'étude du ghetto américain», M: Mensuel Marxisme Mouvement, numéro spécial "Mégalopole USA », 85-86, novembredécembre 1996, p. 36-41] ; Loïc Wacquant, «L'“underclass” urbaine dans l'imaginaire social et scientifique américain », dans L'Exclusion : l'état des savoirs, Paris, Éditions La Découverte, 1996, p. 248-262.

${ }^{34}$ Loïc Wacquant, Parias urbains. Ghetto, banlieues, État, Paris, La Découverte, 2006.
} 


\section{Le corps, le ghetto et l'État pénal}

l'écrasement de l'espace des possibles et le climat de claustration sociale et raciale qui imprègnent le South Side dans les années 1990, dont on ne peut pas se faire idée si on n'y traîne pas ses guêtres. Le schéma de la marginalité avancée, développé dans la troisième partie du livre, caractérisant le nouveau régime de pauvreté qui émerge à l'ère post-keynésienne et post-fordiste, s'appuie sur la connaissance directe des stratégies de vie des habitants du ghetto noir américain et des banlieues françaises en déclin, des formes vivantes de la conscience collective qui orientent leurs actions et leurs aspirations et des obstacles concrets sur lesquelles elles butent - comme l'absence d'un langage commun qui redouble au niveau symbolique la dispersion objective du «précariat ».

Le concept de stigmatisation territoriale comme modalité distinctive du discrédit collectif jeté sur les résidents des quartiers de relégation à l'âge du salariat désocialisé trouve son origine dans l'enquête menée en face-à-face auprès des responsables de la politique de la ville en France. ${ }^{35}$ Les hauts-fonctionnaires que j'interrogeais parlaient tous des quartiers populaires de la périphérie avec des trémolos d'angoisse et de dégoût dans la voix ; tout dans leur ton, leur vocabulaire, leur posture et leur gestuelle, exprimait le regret d'être en charge d'une mission et d'une population avilies et donc avilissantes. Puis j'ai retrouvé le même sentiment de dégoût et d'indignité tout en bas de l'échelle urbaine, parmi les habitants de la cité des Quatre mille en banlieue de Paris comme chez les Noirs américains pris au piège de l'hyperghetto à Chicago. Je n'aurais pas pu développer cette notion qui m'apparaît rétrospectivement comme un des résultats les plus probants de cette recherche - sans le travail de terrain mené en parallèle des deux côtés de l'Atlantique.

Susana Durão - Comment la stigmatisation territoriale se distinguet-elle de la stigmatisation ethnique et en quoi est-elle si importante à vos yeux?

Loïc Wacquant - Les quartiers ouvriers, déshérités ou immigrés n'ont jamais eu bonne réputation, et la ville a toujours eu ses basfonds et ses secteurs interlopes entourés d'une aura sulfureuse. Mais un phénomène nouveau est apparu au cours des deux dernières

\footnotetext{
${ }^{35}$ Loï Wacquant, «Territorial Stigmatization in the Age of Advanced Marginality Thesis Eleven, 91, novembre 2007, p. 66-77.
} 


\section{Labyrinthe, $n^{\circ} 31$}

décennies : dans tous les pays avancés, un petit nombre de quartiers ou de localités sont désormais publiquement connus comme des puits de perdition sociale et morale. Le grand ensemble de Robert Taylor Homes à Chicago, Bobigny à la périphérie de Paris, le district de Moss Side à Manchester, Tensta aux portes de Stockholm, São João de Deus au nord de Porto: ces noms sont autant d'éponymes nationaux de l'«horreur urbaine »; ils inspirent l'effroi et le honnissement dans toute la société. Une flétrissure de lieu s'est cristallisée, qui se surajoute au déshonneur de classe et d'ethnicité frappant déjà leurs habitants, avec des effets propres, distincts des «marques » tribales, morales ou corporelles traitées jadis par Erving Goffman ${ }^{36}$, qui contribuent puissamment à la spirale de la désintégration sociale et de la diffamation symbolique.

Quand je demandais aux habitants du ghetto de Chicago et des cités de La Courneuve, deux zones de relégation distantes de six mille kilomètres, «que font les gens du quartier pour se débrouiller au jour le jour », ils répondaient d'emblée en termes quasi-identiques : «Ah, moi, les gens du quartier, je les connais pas. Je vis ici mais je ne suis pas d'ici»- autrement dit, je ne suis pas comme «eux». Ils se démarquaient de leurs voisins et reportaient sur ces derniers l'image dégradée que donne d'eux le discours public. Des deux côtés de l'Atlantique, les habitants des districts perçus et vécus comme des purgatoires urbains dissimulent leur adresse aux employeurs et aux services publics, évitent de recevoir des amis chez eux et nient appartenir à la microsociété locale. Seule l'enquête de terrain pouvait révéler la prégnance de ce sentiment d'indignité sur les deux sites et le recours aux mêmes stratégies de gestion du stigmate territorial que sont la distanciation mutuelle et le dénigrement latéral, la retraite dans la sphère privée et la fuite à l'extérieur dès qu'on en acquiert les moyens. Ces stratégies tendent à défaire un peu plus les collectifs déjà affaiblis des zones urbaines déshéritées et à produire la «désorganisation» dont le discours dominant dit justement qu'elle caractérise ces zones. Le stigmate territorial incite également l'État à adopter des politiques spécifiques, dérogatoires du droit commun et de la norme nationale, qui le plus souvent renforcent la dynamique de

\footnotetext{
${ }^{36}$ Erving Goffman, Stigma : Notes on the Management of Spoiled Identity, New York, Simon \& Schuster, 1963
} 
marginalisation qu'elles prétendent combattre, au détriment des habitants.

\section{LA CHAIR ET LE TEXTE}

Susana Durão - Du point de vue de la méthode, de l'échelle et de l'objet, l'ethnographie du métier de boxeur à Woodlawn est très différente. Comme s'est-elle déroulée?

Loïc Wacquant - C'est une ethnographie de facture classique par ses paramètres, une sorte d'étude de village comme en faisait l'anthropologie britannique dans les années 1940, à ceci près que mon village est la salle de boxe et ses extensions, et ma tribu les boxeurs et leur entourage ${ }^{37}$. J'ai retenu cette unité structurale et fonctionnelle parce qu'elle enserre les boxeurs et découpe un horizon temporel, relationnel, mental, émotionnel et esthétique spécifique, qui sépare le pugiliste et l'élève au dessus de son environnement ordinaire. J'ai voulu d'abord disséquer la relation bifide d' "opposition symbiotique » entre le ghetto et le gym, la rue et le ring; puis montrer comment la structure sociale et symbolique de la salle gouverne la transmission de la technique du Noble art et la production de la croyance collective dans l'illusio pugilistique; et, enfin, percer la logique pratique d'une pratique corporelle à la limite de la pratique par le biais d'un apprentissage de longue durée à la première personne. Pendant trois ans, je me suis fondu dans le paysage local et pris au jeu. J'ai appris à boxer et participé à toutes les phases de la préparation du boxeur, jusqu'à combattre lors du grand tournoi des Golden Gloves. J'ai suivi mes copains de la salle dans leurs pérégrinations personnelles et professionnelles. Et j'ai traité au quotidien avec les entraîneurs, managers, promoteurs, etc., qui font tourner la planète de la cogne. Ce faisant, j'ai été aspiré par la spirale sensuelle et morale du pugilisme, au point d'envisager d'interrompre ma trajectoire universitaire pour passer professionnel.

C'est dire que l'objet et la méthode de cette enquête, elles, ne sont pas classiques. Corps et âme offre une radicalisation empirique et

\footnotetext{
${ }^{37}$ Loï Wacquant, Corps et âme. Carnets ethnographiques d'un apprenti boxeur,
} Marseille et Montréal, Agone, Comeau \& Nadeau, 2000. 


\section{Labyrinthe, $n^{\circ} 31$}

méthodologique de la théorie de l'habitus de Bourdieu ${ }^{38}$. D'une part, j'ouvre la «boîte noire » de l'habitus pugilistique en décortiquant la production et l'assemblage des catégories cognitives, des habiletés corporelles et des désirs qui, combinés, définissent la compétence et l'appétence propres au boxeur. De l'autre, je déploie l'habitus comme dispositif méthodologique, c'est-à-dire que je me mets en situation d'acquérir par la pratique, en temps réel, les dispositions du boxeur, afin d'élucider le magnétisme propre cosmos pugilistique. La méthode met donc à l'épreuve la théorie de l'action qui anime l'analyse selon un dispositif de recherche récursif et réflexif.

L'idée qui me guidait ici, c'est de pousser la logique de l'observation participante jusqu'à inverser cette dualité et faire de la participation observante. Dans la tradition anglo-américaine, on dit aux étudiants d'anthropologie, quand ils s'initient au travail de terrain: "Don't go native ». Dans la tradition française, on peut admettre l'immersion radicale - ainsi Jeanne Favret-Saada dans Les mots, la mort, les sorts ${ }^{39}$ - mais à condition qu'elle soit couplée avec une épistémologie subjectiviste qui nous perd dans le fors intérieur de l'anthropologue-sujet. Moi je dis au contraire, "Go native », mais "go native armed», soit équipé de tous vos outils théoriques et méthodologiques, avec toutes les problématiques héritées de votre discipline, avec votre capacité de réflexivité et d'analyse, et guidé par un effort constant pour, après être passé par l'épreuve initiatique, objectiver cette expérience et construire l'objet - plutôt que de se laisser naïvement embrasser et construire par lui. Allez-y, devenez un indigène, mais revenez en sociologue !

\footnotetext{
${ }^{38}$ Loïc Wacquant, «Taking Bourdieu into the Field », Berkeley Journal of Sociology, 46, 2002, p. 180-186 [trad. française : «Le pari pascalien de Bourdieu », dans Gérard Mauger (éd.), Rencontres avec Pierre Bourdieu, Paris, Éditions du Croquant, 2005, p. 271-279)] ; et «Habitus », dans Jens Beckert et Milan Zafirovski (dir.), International Encyclopedia of Economic Sociology, Londres, Routledge, 2004, p. 315-319.

${ }^{39}$ Jeanne Favret-Saada, Les Mots, la mort, les sorts, Paris, Gallimard/Poche, 1985.
} 


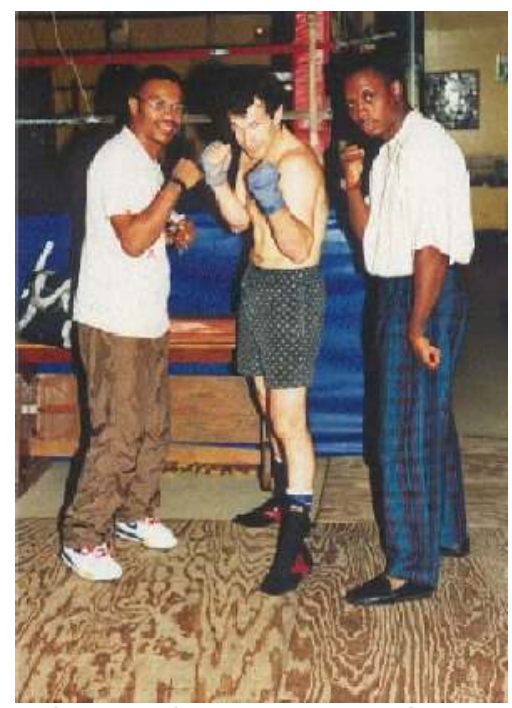

« Busy Louie » à l'entraînement, flanqué de ses partenaires Curtis et Tony (mai 1990) [coll. de l'auteur]

Susana Durão - C'est cette initiation guidée par la théorie qui fait l'originalité de Corps et âme, à en juger par les nombreuses réactions qu'a suscitées le livre (traduit en neuf langues et très largement recensé au-delà de la sociologie).

Loïc Wacquant - Sur les réactions, je n'en suis pas sûr! Je crois, à mon grand regret puisque l'intention majeure de l'enquête est de désexotiser le métier de la cogne, que le retentissement du livre tient pour partie au côté «sensationnel» du travail de terrain: se faire casser le nez pour comprendre ce que c'est que de devenir boxeur, ce n'est pas commun, encore moins si c'est un petit Blanc français qui s'y colle dans le ghetto noir américain... Certains de mes critiques, se méprenant sur mon travail en y voyant une extension des «études de profession » à la manière de la seconde École de Chicago, n'ont pas même aperçu le double rôle que joue le concept d'habitus dans l'enquête et se sont plaints de l'absence de théorie dans le livre ${ }^{40}$ !

${ }^{40}$ Loï Wacquant, «Shadowboxing with Ethnographic Ghosts: A Rejoinder», Symbolic Interaction, 28-3, Summer 2005, p. 441-447 (réponse au symposium sur Body and Soul). 


\section{Labyrinthe, $n^{\circ} 31$}

En fait, la théorie et la méthode sont jointes au point de fusionner dans l'objet empirique même qu'elles permettent d'élaborer. Corps et âme est une ethnographie expérimentale au sens originel du terme, puisque le chercheur est l'un des corps socialisés jetés dans l'alambic sociomoral et sensuel de la salle de boxe, corps en action dont on va tracer la trans-mutation pour percer l'alchimie par laquelle se fabrique le boxeur. L'apprentissage est ici le moyen d'acquérir une maîtrise pratique, une connaissance viscérale de l'univers en question, de pénétrer la praxéologie des agents en question et non pas d'entrer dans la subjectivité du chercheur. Ce n'est pas du tout une chute dans le puits sans fond du subjectivisme, dans lequel se lance l'«autoethnographie », au contraire : c'est s'appuyer sur l'expérience la plus intime, celle du corps désirant qui souffre, pour saisir in vivo l'usinage collectif des schèmes de perception, d'appréciation et d'action pugilistiques qui sont partagées, peu ou prou, par tous les boxeurs, quels que soient leur origine, leur trajectoire et leur niveau dans la hiérarchie sportive ${ }^{41}$. Le personnage central de l'histoire, ce n'est ni Busy Louie, ni tel ou tel boxeur, ni même DeeDee le vieux coach, en dépit de sa position de chef d'orchestre : c'est le gym en tant que forge sociale et morale. Le modèle intellectuel, ce n'est pas Castañeda et ses sorciers Yaqui mais le Bachelard du Rationalisme appliqué et de la poétique matérialiste de l'espace, du temps et du feu ${ }^{42}$.

En fait, je pense que j'ai fait de manière explicite, méthodique et surtout extrême, ce que fait tout bon ethnographe: se donner une appréhension pratique, tactile, sensorielle de la réalité prosaïque qu'elle étudie de sorte à élucider les catégories et les relations qui organisent le comportement et les sentiments ordinaires de ses sujets. Sauf que d'habitude on le fait sans le dire, ou sans thématiser le rôle de la «co-présence » au phénomène, ou en (se) faisant croire que c'est un processus mental et non un apprentissage corporel et sensuel qui procède en deçà de la conscience avant de passer par la médiation du langage. Corps et âme apporte la démonstration en actes des

\footnotetext{
41 Loïc Wacquant, «Carnal Connections : On Embodiment, Membership, and Apprenticeship », Qualitative Sociology, 28-4, hiver 2005, p. 445-471 (réponse au numéro spécial consacré à Body and Soul, 28-3, automne 2005).

${ }^{42}$ Gaston Bachelard, Le Rationalisme appliqué, Paris, PUF, 1949 ; La Psychanalyse du feu, Paris, Gallimard, 1938 ; La Poétique de l'espace, Paris, PUF, 1957.
} 


\section{Le corps, le ghetto et l'État pénal}

possibilités et des vertus distinctives d'une sociologie charnelle ${ }^{43}$, qui tient pleinement compte du fait que l'agent social est un animal qui souffre, un être de chair et de sang, de nerfs et de viscères, habité par des passions et doté de savoirs et d'habiletés incorporées - par opposition à l'animal symbolicum de la tradition néo-kantienne, reprise par Clifford Geertz et les tenants de l'anthropologie interprétative, d'un côté, et par Herbert Blumer et l'interactionnisme symbolique, de l'autre ${ }^{44}$ - et que cela est vrai aussi du sociologue. Cela implique de remettre le corps du sociologue en jeu et de traiter son organisme intelligent, non pas comme un obstacle au savoir, comme le voudrait l'intellectualisme vrillé à la conception indigène de la pratique intellectuelle, mais comme vecteur de connaissance du monde social.

Susana Durão - Mais alors, si Corps et âme ne se centre pas sur la personne de Busy Louie, le sociologue-boxeur en apprentissage, estce à dire que ce n'est pas un livre d'anthropologie réflexive?

Loïc Wacquant - Pas au sens où l'entend l'anthropologie dite « poststructuraliste » ou « postmoderne », pour qui le retour du regard analytique est dirigé, soit sur le sujet connaissant dans son intimité personnelle, soit sur le texte qu'elle livre à ses pairs et les circuits de pouvoir-savoir dans lesquels il circule. Ces formes de réflexivité, narcissique et discursive, sont très superficielles; elles peuvent certes être un moment utile dans la recherche pour limiter le jeu des biais les plus grossiers (identité et trajectoire sociales, affects, effets rhétoriques, etc.). Mais elles arrêtent le mouvement de l'auto-critique là où il devrait commencer, soit par la mise en question continuelle des catégories et des techniques d'analyse sociologique et du rapport au monde qu'elles présupposent. C'est ce retour sur les instruments de construction de l'objet, par opposition au sujet de l'objectivation, qui est la marque distinctive de ce que l'on peut appeler la réflexivité épistémique ${ }^{45}$. Autre différence avec la réflexivité «égologique» ou

\footnotetext{
${ }^{43}$ Loïc Wacquant, «Une expérience de sociologie charnelle», Solidarités, 29, juin 2003, p. $18-20$

${ }^{44}$ Clifford Geertz, The Interpretation of Cultures, New York, Basic Book, 1974 [trad. française : L'Interprétation des cultures, Paris, Gallimard, 1983] ; Herbert Blumer, Symbolic Interaction, Englewood Cliffs, Prentice-Hall, 1966.

${ }^{45}$ Pierre Bourdieu et Loïc Wacquant, An Invitation to Reflexive Sociology, Chicago, University of Chicago Press, 1992, p. 36-46; Pierre Bourdieu, «Participant
} 


\section{Labyrinthe, $n^{\circ} 31$}

textuelle des anthropologues subjectivistes : elle se déploie non pas en fin de projet, ex post, au moment de rédiger son compte-rendu, mais durante, à toutes les étapes de l'investigation. Elle vise l'ensemble des opérations les plus routinières, depuis la sélection du site et des informateurs jusqu'aux questions à poser ou à éviter, en passant par l'engagement des schèmes théoriques, des outils méthodologiques et des techniques d'exposition, au moment où elles sont effectuées.

Corps et âme est donc un livre réflexif au sens où le dispositif même de l'enquête me forçait en permanence à réfléchir sur l'adéquation des moyens d'investigation à leur fin, sur la différence entre la maîtrise pratique et la maîtrise théorique d'une pratique, sur la marge entre l'engouement sensoriel et la compréhension analytique, le viscéral et le mental, l'ethos et le logos du pugilisme mais aussi de la sociologie. De même, Parias urbains est un ouvrage de sociologie urbaine réflexive parce qu'il questionne sans cesse les catégories mêmes qu'il met en cause et en jeu - " underclass », " inner city », « banlieues », hyperghetto, relégation, précariat - pour penser les nouvelles configurations de la marginalité dans la ville. Et parce qu'il s'appuie sur une démarcation claire entre catégories indigènes et catégories analytiques, qui est pour moi le socle de la réflexivité.

La réflexivité épistémique s'impose avec une urgence d'autant plus grande à l'ethnographe que tout l'invite à se soumettre aux préconstructions du sens commun. Par devoir méthodologique, elle se doit d'être à l'écoute des agents qu'elle étudie et de prendre au sérieux leur " point de vue». Si elle fait bien son travail, elle se trouve liée à eux par des rapports affectifs qui encouragent l'identification et le transfert. Enfin l'image publique de l'ethnographie (y compris chez les autres chercheurs en science sociale, malheureusement) la rapproche du récit, du journal intime, voire de l'épopée. C'est pourquoi l'anthropologue ou le sociologue qui recourt au travail de terrain se doit de redoubler de réflexivité. C'est ce que j'essayé de montrer dans «Scrutinizing the Street » à propos de dérives récentes dans l'ethnographie urbaine états-unienne ${ }^{46}$. L'objet bien considéré de ma critique n'est pas les trois livres que je passe au crible analytique

Objectivation : The Huxley Medal Lecture ", Journal of the Royal Anthropological Institute, 9-2, février 2002, p. 281-294 [trad. française : « L'objectivation participante », Actes de la recherche en sciences sociales, $\mathrm{n}^{\circ} 150$, septembre 2003, p. 43-57].

${ }^{46}$ Loïc Wacquant, « Scrutinizing the Street : Poverty, Morality, and the Pitfalls of Urban Ethnography », American Journal of Sociology, 107-6, mai 2002, p. 1468-1532. 


\section{Le corps, le ghetto et l'État pénal}

(et encore moins leurs auteurs, qui ne sont ici que des points dans l'espace académique, ou leurs options politiques, qui me sont indifférentes), mais une certaine posture épistémologique d'abandon irréfléchi aux aperceptions indigènes, au moralisme ordinaire, aux séductions de la pensée officielle et aux règles de la bienséance académique. Cette posture est au principe d'erreurs scientifiques graves, parce qu'elles font système et qu'elles ont le sens commun ordinaire et le sens commun savant pour elles.

Susana Durão - Corps et âme innove aussi dans la forme, par son écriture narrative de facture quasi théâtrale qui invite le lecteur à vibrer avec l'apprenti-boxeur et qui donne à voir à la fois la logique du travail de terrain et son produit.

Loïc Wacquant - Comment passer des tripes à l'intellect, de la compréhension de la chair au savoir du texte ? Voilà un vrai problème d'épistémologie concrète sur lequel on n'a pas suffisamment réfléchi, et qui m'a longtemps semblé insoluble. Restituer la dimension charnelle de l'existence ordinaire et l'ancrage corporel du savoir pratique constitutif du pugilisme - mais encore de toute pratique, même les moins «corporées » en apparence - requiert en effet un remaniement profond de notre manière de rédiger la science sociale. Dans le cas présent, il me fallait trouver un style en rupture avec l'écriture monologique, monochrome, linéaire, d'un compte-rendu classique duquel l'ethnographe s'est retiré, pour mettre au point une écriture à plusieurs facettes, mêlant les styles et les genres, afin de capturer et de transmettre au lecteur «la douleur et la saveur de l'action $»^{47}$.

Corps et âme est écrit contre le subjectivisme, contre le narcissisme et l'irrationalisme qui sous-tendent certaines théories littéraires dites "postmodernes", mais ça ne veut pas dire qu'il faut pour autant se priver des techniques littéraires et des instruments d'exposition dramatique que nous donne cette tradition. C'est pourquoi le livre mêle trois formes d'écriture qui, tout en s'entrecroisant au fil des pages, se partagent la priorité dans les trois

\footnotetext{
${ }^{47}$ Loïc Wacquant, « O sabor e a dor da ação », Prefácio de Corpo e alma, op. cit., 2002, p. 11-17. (Cette préface théorique, essentielle à la compréhension du livre, a malheureusement été exclue de la deuxième édition française de Corps et âme parue en 2002, à l'insistance de son éditeur; on en trouvera une variante dans le texte « $\mathrm{La}$ saveur et la douleur de l'action », Corps et culture, 6, printemps 2004, pp. 257-261).
} 


\section{Labyrinthe, $n^{\circ} 31$}

parties, de sorte que le lecteur glisse insensiblement du concept au percept, de l'analyse à l'expérience. La première partie ancre une écriture sociologique classique de type analytique qui isole d'entrée les structures et les mécanismes de façon à donner au lecteur les instruments nécessaires pour expliquer et comprendre ce qui se passe. Le ton de la seconde partie est donné par une écriture ethnographique stricto sensu, c'est-à-dire dépictive des manières d'être, de penser, de sentir et d'agir propres au milieu considéré, où l'on retrouve ces mêmes mécanismes mais en action, à travers leurs produits. Avec la troisième partie vient le moment expérientiel, sous la forme d'une «nouvelle sociologique » qui livre le ressenti, l'expérience vécue du sujet qui se trouve être aussi l'analyste.

La combinaison raisonnée de ces trois modalités d'écriture sociologique, ethnographique et littéraire - selon des proportions qui vont en s'inversant au fil des pages, vise à permettre au lecteur à la fois de ressentir émotionnellement et de comprendre rationnellement les ressorts et les tours de l'action pugilistique. Pour cela, le texte tresse une trame analytique, des plages de notes de terrain soigneusement éditées, des contrepoints faits de portraits de personnages-clefs et d'extraits d'interviews, et des photographies dont le rôle est de favoriser une aperception synthétique du jeu dynamique des facteurs et des formes répertoriées dans l'analyse, de donner à « toucher avec les yeux » le pouls battant du pugilisme. Là encore, la théorie de l'habitus, le recours à l'apprentissage comme technique d'investigation, la place accordée au corps sensible comme vecteur de connaissance et l'innovation formelle dans l'écriture : tout se tient. Il ne sert à rien de faire une sociologie charnelle adossée à une initiation pratique si ce qu'elle révèle du magnétisme sensorimoteur de l'univers en question disparaît ensuite à la rédaction, sous prétexte qu'il faut respecter des canons textuels dictés par le positivisme ou le cognitivisme néo-kantien.

\section{EN BOBSLEIGH À TRAVERS L'ATLANTIQUE}

Susana Durão - Venons-en à votre position de sociologue européen qui travaille aux États-Unis : l'occasion de réfléchir à la conception de l'intellectuel en cours de chacun des deux côtés de l'Atlantique.

Loïc Wacquant - Je suis à la charnière - ou en porte-à-faux - entre 


\section{Le corps, le ghetto et l'État pénal}

deux traditions, deux conceptions du travail de recherche. Il y a d'un côté une tradition européenne, incarnée par la France, qui a inventé la catégorie sociohistorique de l'intellectuel au moment de l'affaire Dreyfus, comme l'a bien montré Christophe Charle dans son magnifique livre Naissance de l'intellectuel. Pour ce lignage qui court, en gros, de Zola à Sartre, puis de Foucault à Bourdieu et d'autres, l'intellectuel est un producteur culturel qui par définition engage sa compétence propre dans le débat public ${ }^{48}$. Nécessairement impliqué dans la Cité, il est de son devoir de réinjecter dans la sphère civique et politique le fruit de ses réflexions et de ses observations. Je suis un produit de cette tradition.

Mais il se trouve que je conduis mes travaux principalement de l'autre côté de l'Atlantique, où règne une autre tradition, plus soucieuse de rigueur méthodologique, pour laquelle l'idéal du chercheur est incarné non pas par l'intellectuel (c'est un mot péjoratif aux États-Unis), mais par le professional, au sens de l'avocat ou du médecin, soit le porteur d'une compétence technique et d'un savoir expert qui est un savoir neutre, qui ne doit être jugé que par les pairs et qui pour cela doit se tenir à l'écart du débat public. L'intellectuel est bidimensionnel, à la fois savant et citoyen actif; l'academic est unidimensionnel, tourné exclusivement vers le microcosme universitaire - sous peine de se voir discréditer. C'est pour moi une tension professionnelle et existentielle qui n'est pas toujours facile à gérer.

Bien sûr, chacune de ces traditions a ses vertus et ses travers propres. Plutôt que de s'enfermer dans la célébration rituelle d'une conception de la vocation de chercheur et le dénigrement de l'autre, il faut s'efforcer de cumuler leurs qualités distinctives. La force du patron américain réside dans la rigueur méthodologique qu'il prescrit et qui freine, voire interdit l'amateurisme. La perversion du patron français, à l'inverse, est la grande tolérance envers le dilettantisme intellectuel et à l'essayisme à prétention philosophique - incarné jusqu'à la caricature par ce que Louis Pinto appelle l'«intellectuel médiatique ", qui n'existe que par et pour les médias ${ }^{49}$. Combien de nos grands «philosophes » parisiens omniprésents dans les magazines

\footnotetext{
${ }^{48}$ Christophe Charle, Naissance des « intellectuels » (1880-1900), Paris, Éditions de Minuit, 1990.

${ }^{49}$ Louis Pinto, La Vocation et le métier de philosophe. Pour une sociologie de la philosophie dans la France contemporaine, Paris, Seuil, 2007.
} 
culturels et sur les plateaux de télévision, n'ont jamais publié le moindre travail dans une revue sérieuse de philosophie ou de science sociale. Mais tant que leurs amis journalistes parisiens s'émerveillent de leurs fortes pensées, ils existent en tant que tel... Sur les deux rives de l'Atlantique, les chercheurs autonomes sont de plus en plus doublés par les experts bureaucratiques, savants de l'ombre qui apportent aux gouvernants les réponses qu'ils souhaitent, et surtout qui acceptent leurs questions. En fait, dans tous les pays, il y a un gros déficit de réflexion collective sur l'organisation collective du travail scientifique et sur les rapports changeants entre la recherche, les médias, l'argent et la politique. C'est ce déficit qui favorise l'hétéronomie scientifique et, partant, la diffusion de la «pensée unique » néolibérale qui tronque et paralyse le débat public depuis une décennie ${ }^{50}$.

Susana Durão - Vous partagez votre temps entre les États-Unis et l'Europe, mais à quoi concrètement ressemble la vie quotidienne de Loïc Wacquant?

Loïc Wacquant - Il ne vaut mieux pas essayer de la décrire ! C'est plutôt frénétique... un peu comme de faire du bobsleigh, où on est allongé au ras du sol dans un bolide qui descend la montagne à vitesse vertigineuse. Voilà, la vie quotidienne de Loïc Wacquant, c'est faire $\mathrm{du}$ bobsleigh intellectuel mais sans la glace [rires]... Juste une confidence : il est rare que je me couche tôt et je ne vais pas souvent au cinéma.

Ma vie quotidienne est semblable à celle de tous les chercheurs. Quand je suis aux États-Unis, pour mon enseignement et sur mon terrain principal, j'ai une vie d'hermite où je suis assez enfermé, isolé dans le monde universitaire qui est lui-même totalement isolé de la société environnante et structuralement déconnecté du champ politique. Donc je me concentre sur mes recherches, mes cours, le suivi des doctorants, la rédaction d'Ethnography, etc. C'est 95\% de mon temps et de mon énergie, et c'est la base de tout : sans travail scientifique sérieux, sans connaissance mesurée et éprouvée, je n'aurais rien à dire. C'est quand je viens en Europe que le côté « intellectuel engagé » (comme disent mes collègues américains) ressort. Une fois traversé l'Atlantique, je donne des conférences et je

\footnotetext{
${ }^{50}$ Pierre Bourdieu et Loïc Wacquant, «Les ruses de la raison impérialiste », Actes de la
} recherche en sciences sociales, 121-122, mars 1998, p. 109-118. 
participe à des colloques scientifiques, mais je prend aussi part de temps à autre à des événements extra-universitaires, à des débats publics qui sont l'occasion d'intervenir sur les sujets où $\mathrm{j}$ 'ai une compétence. Heureusement que cette possibilité de sortir du bocal universitaire existe en Europe et en Amérique latine, sinon je crois que je serais complètement desséché humainement et intellectuellement! Quand on reste enfermé dans le cénacle universitaire, on se laisse prendre par les jeux et les enjeux du microcosme, et au final on perd son énergie civique, sa capacité à l'étonnement devant le monde et son habileté à le décrypter.

J'ai parfois le sentiment d'avoir une existence dédoublée ou démultipliée, avec des moments de tension entre les différents sujets que je creuse, entre le plan scientifique et le plan politique, entre les publics universitaires et militants, entre les États-Unis et l'Europe, où, comme je l'ai dit, prévalent des conceptions différentes de l'activité intellectuelle et donc des images assez différentes de mon travail. Parfois, ces deux dimensions se télescopent et c'est difficile, voire douloureux ; mais quand elles rentrent en synergie, là j'ai le sentiment de faire mon ouvrage à plein. Un exemple : en février dernier, au moment où la campagne présidentielle démarrait en France, j'ai participé à un débat public autour de Parias urbains organisé par Utopia, un groupe de militants de gauche «transcourants", où le discutant du livre était l'ancien ministre de l'intérieur de la gauche plurielle, Jean-Pierre Chevènement, que $\mathrm{j}$ 'avais éreinté dans mon livre précédent, Les Prisons de la misère. C'était une discussion aussi sérieuse que celle que j'avais eu la semaine d'avant avec des collègues britanniques sociologues et urbanologues à Cambridge, mais plus ouverte et plus risquée. C'était très stimulant de confronter nos visions de la marginalisation des banlieues populaires et des remèdes que l'État pourrait y apporter, de réfléchir à haute voix aux manières possibles de prolonger mes analyses en mesures pratiques, sans rien céder en rigueur théorique et empirique ${ }^{51}$.

En France ou au Portugal, en Argentine, au Mexique ou en Belgique, quand je donne des conférences, le public est souvent un assemblage bigarré d'universitaires, de militants politiques, de défenseurs des droits de l'homme, de gens provenant de milieux professionnels divers comme les enseignants, les travailleurs sociaux,

\footnotetext{
${ }^{51}$ La vidéo est en ligne à cette adresse $: \underline{h t t p: / / u t o p i a c o n f . f r e e . f r / v i d e o . h t m . ~}$
} 
et des citoyens ordinaires. Cela donne la possibilité d'avoir un dialogue plus large et plus ouvert, où le regard et le langage savants sont eux-mêmes questionnés et où se pose le problème de retraduire en termes civiques et pratiques les résultats des travaux que l'on a conduits dans un cadre proprement scientifique.

Susana Durão - Et cela n'est pas possible au États-Unis ?

Loïc Wacquant - Cet espace de «traduction» collective est extrêmement réduit du fait du verrouillage du champ politique et de l'auto-enfermement des professions universitaire. Par exemple, alors qu'en Europe ou en Amérique latine, je suis intervenu cent fois sur la question de l'emprisonnement à la radio, à la télévision, dans les grands quotidiens, et que j'ai été consulté par les hauts responsables administratifs ou politiques, aux États-Unis je n'ai jamais donné une seule conférence sur les prisons hors du cadre universitaire, où le public-type est composé presqu'exclusivement d'étudiants et de professeurs, sociologues, criminologues ou juristes. Et ce n'est pas une carence personnelle : c'est vrai de tous les chercheurs en pointe, comme mon éminent collègue le juriste Franklin Zimring, qui a produit une étude fracassante sur la politique de «perpétuité pour double récidive » en Californie montrant l'absurdité juridique et criminologique de la loi dite "Three Strikes and You're Out»" ${ }^{52}$. Pas un responsable politique ou administratif de l'État n'a jamais daigné le consulter sur le sujet. Et la Californie dépense milliards de dollars chaque année pour enfermer 170000 condamnés, trois fois le stock carcéral de la France...

Il n'existe pratiquement pas de lieu de discussion civique et de véhicule organisationnel pour faire passer le travail scientifique dans la sphère publique et lui donner un poids. Il y a bien une nébuleuse de " community organizations" mais elles occupent une position marginale dans le champ bureaucratique - elles sont même, à mon sens, un instrument de domestication de la contestation politique. Autre obstacle sur la voie de la mise en valeur civique du travail scientifique, les public policy schools et les think tanks privés qui servent de glacis intellectuel ou de «bouclier» protégeant les

\footnotetext{
${ }^{52}$ Franklin E. Zimring, Gordon Hawkins et Sam Kamin, Punishment and Democracy: Three Strikes and You're Out in California, New York, Oxford University Press, 2001.
} 
décideurs politiques de la pensée critique en produisant un pseudo-savoir préformaté, en tous points conforme aux intérêts des dominants ${ }^{53}$.

Les champs politique et médiatique étatsuniens sont largement contrôlés par les intérêts d'argents, les grandes entreprises et les grosses fortunes qui tiennent sous leur coupe les deux partis siamois, qui ne sont eux-mêmes que des labels pour faciliter la collecte des fonds et payer les campagnes électorales qui exigent de passer par des médias payants. Dit comme ça, cela peut paraître caricatural, mais c'est la réalité qui est caricaturale : pensez que tous les sénateurs sans exception sont millionnaires et que la campagne présidentielle de 2008 va coûter 5 milliards de dollars! En fait, c'est toute l'organisation de la sphère publique étatsunienne qui joue contre l'implication des universitaires dans la vie de la Cité, mais aussi la morale professionnelle des chercheurs qui se voient comme des academics plutôt que comme des intellectuels (qui sentent le souffre et font peur aux deans).

C'est pourquoi je profite d'avoir des attaches professionnelles et personnelles des deux côtés de l'Atlantique pour revenir régulièrement en Europe, où je passe quatre à cinq mois par an. Cela fouette mes énergies et me redonne l'envie de continuer mon travail dans les périodes de doute ou d'épuisement. On a tant à apprendre en sortant de l'«académie» et en restant au contact des gens qui sont directement impliqués au niveau quotidien, professionnel, militant ou politique, au cœur des phénomènes qu'on étudie. En retour, on peut les aider à voir leur propre pratique sous un angle nouveau et parfois à mieux cibler leur action.

\section{LA SCIENCE SOCIALE COMME PHARE ET COMME DISSOLVANT}

Susana Durão - Vos recherches peuvent-elles aider à guider les militants dans leur action?

Loïc Wacquant - C'est à eux de le dire, ou de le découvrir, pas à moi. Mais l'activité militante est pleine de chausse-trapes et de leurres qui mènent à un gaspillage phénoménal des énergies. Quand c'est le cas,

\footnotetext{
53 Loï Wacquant, «Critical Thought as Solvent of Doxa», Constellations, 11-1, printemps 2004, p. 97-101.
} 
il faut avoir l'honnêteté de dire: «Stop, ce n'est pas la bonne question, vous perdez votre temps ». Cela peut être le rôle du chercheur.

Soit un exemple précis : aux États-Unis, les militants pour la justice sont très mobilisés contre la privatisation des prisons et ce que la thèse $\mathrm{du}$ "prison industrial complex" présente comme l'exploitation de la main d'œuvre captive des détenus. En réalité, l'emploi carcéral par des firmes privées concerne à peine $0,3 \%$ des prisonniers : c'est un phénomène absolument minuscule. Batailler pour l'abolition du «travail esclavagiste » en prison, c'est ferrailler avec une chimère. Et si demain on supprimait les prisons commerciales aux États-Unis, le stock carcéral serait inchangé; simplement, on le caserait avec $6 \%$ de cellules en moins. En se focalisant sur la privatisation, on passe à côté de l'essentiel : ce n'est pas la quête du profit capitaliste qui commande l'expansion stupéfiante de la population sous les verrous aux États-Unis mais la construction d'un État libéral-paternaliste, c'est-à-dire un projet proprement politique qui requiert de penser ensemble dérégulation économique, restriction des aides sociales et expansion du secteur pénal $^{54}$. Le même raisonnement vaut pour ce que certains militants de gauche en France appellent naïvement « le programme sécuritaire ». Ce programme n'existe que dans leur imagination: la sécurité n'est qu'un leurre qui détourne le regard du vrai enjeu, qui est le redécoupage du périmètre et des missions de l'État face au Moloch du marché.

Idem à travers l'Europe à propos de la panique morale de la « ghettoïsation» des banlieues populaires : les militants qui s'agitent pour «casser les ghettos» se trompent de cible. Les quartiers marginalisés de la périphérie urbaine d'Europe sont aux antipodes du ghetto. Leurs populations sont mélangées et de plus en plus hétérogènes ethniquement; leurs capacités d'organisation collective vont en diminuant; leurs frontières sont poreuses, et ils sont incapables de produire une identité collective, autre que territoriale et négative. Ce sont des anti-ghettos qui souffrent d'abord de paupérisation et du retrait généralisé de l'État. Au lieu de « seconde génération de l'immigration », il faudrait s'attaquer à la troisième

\footnotetext{
${ }^{54}$ Loïc Wacquant, Punishing the Poor. The New Government of Social Insecurity, Durham and London, Duke University Press, 2008.
} 


\section{Le corps, le ghetto et l'État pénal}

génération du chômage de masse et de la précarité salariale rampante, qui fait saillir la discrimination parce que le marché du travail s'est rétracté et fragmenté ${ }^{55}$.

Susana Durão - Vos analyses donnent parfois le sentiment sombre que le monde social est très hermétique et habité par une causalité implacable; alors, pour finir, quel serait votre message d'optimisme pour l'avenir des sciences sociales?

Loïc Wacquant - Le sociologue n'a à être ni optimiste ni pessimiste ; il doit regarder la réalité en face, avec lucidité et en utilisant tous les instruments que lui apportent sa science. Il doit simplement - mais c'est plus simple à dire qu'à faire ! - être rigoureux et intrépide dans l'analyse pour construire un modèle vrai qui permette d'identifier les points d'intervention et les leviers possibles d'une intervention individuelle et collective ${ }^{56}$. Si mes analyses sont souvent sombres et froides, c'est parce que l'époque est sombre et froide ! Ce n'est pas un trait de caractère de l'analyste mais une propriété de la réalité historique. Maintenant, c'est sûr que si on regarde le monde avec les yeux des dominants, le paysage social est beaucoup plus rose et enthousiasmant!

Cela dit, les sciences sociales d'aujourd'hui peuvent apporter une contribution civique de premier plan, en jouant le double rôle de dissolvant et de phare. Dissolvant du nouveau sens commun néolibéral qui «naturalise » l'état actuel du monde et ses tendances immanentes, par la critique méthodique des catégories et des topiques qui tissent le discours dominant. ${ }^{57}$ Il s'agit ici de donner au plus grand nombre de citoyens possible des instruments de réflexion pour se réapproprier leur propre pensée du monde social, pour qu'ils ne soient pas pensés par les médias, habités par les idées préfabriquées que ces derniers diffusent à flux tendus, qu'ils puissent questionner les

\footnotetext{
${ }^{55}$ Loïc Wacquant, «Ghettos and Anti-Ghettos: An Anatomy of the New Urban Poverty », Thesis Eleven, Spring 2008.

${ }^{56}$ Sur le front pénal voir Loïc Wacquant, «L'avènement de l’État pénal n'est pas une fatalité ", Dedans-Dehors (Observatoire international des prisons), 17, janvier-février 2001, p. 16-17 [http://astree.ifrance.com/paroles.htm, page consultée le 30 août 2008] ; «Socialiser, médicaliser, pénaliser : un choix politique », Combats face au sida. Santé, drogues, société, 27, mars 2002, p. 4-9; "Comment sortir du piège sécuritaire», Contradictions (Bruxelles), 22, décember 2004, p. 120-133. Sur le front urbain : Loïc Wacquant, «Ghetto, banlieues, État : réaffirmer la primauté du politique », Nouveaux regards, 33, avril-juin 2006, p. 62-66.

${ }^{57} \lll$ Critical Thought as Solvent of Doxa », art. cit.
} 


\section{Labyrinthe, $n^{\circ} 31$}

schèmes du débat politique - de façon à remettre en cause non seulement les solutions proposées mais le diagnostic même des problèmes que la société confronte. La science sociale peut aussi fonctionner à la manière d'un phare qui éclaire les transformations contemporaines, faisant émerger de l'ombre des propriétés latentes ou des tendances inaperçues (un exemple simple: la vitesse de l'augmentation de l'indice de Gini qui mesure l'inégalité des revenus), et surtout qui révèle des possibles latéraux, des points de bifurcation possible dans le cheminement de l'histoire.

Contre la mythologie de la «mondialisation », doux nom que s'est donnée la révolution néolibérale, les sciences sociales peuvent et doivent réinsuffler dans le débat public l'idée qu'il existe des variations sociologiques très significatives parmi les sociétés contemporaines, qu'on présente abusivement comme uniformisées et contraintes de s'aligner toutes sur le modèle de la «société d'insécurité avancée » incarnée par les États-Unis ou sur leur succursale pratique et idéologique qu'est devenue l'Angleterre. Ces variations sont le résultat agrégé de choix politiques que nous devons faire, non pas dans le noir et à tâtons, mais à la lumière des sciences de la société, en pleine connaissance de cause et de conséquences.

Transcription et révision initiale par Élisabeth Coutant Recherche bibliographique par Susana Durão (complétée, pour les références françaises, par Grégoire Leménager) 